\title{
Bi-unitary multiperfect numbers, III
}

\section{Pentti Haukkanen $^{1}$ and Varanasi Sitaramaiah ${ }^{2}$}

\author{
${ }^{1}$ Faculty of Information Technology and Communication Sciences \\ FI-33014 Tampere University, Finland \\ e-mail: pentti.haukkanen@tuni.fi \\ 2 1/194e, Poola Subbaiah Street, Taluk Office Road, Markapur, Prakasam District, \\ Andhra Pradesh, 523316 India \\ e-mail: sitaramaiah52@gmail.com
}

Dedicated to the memory of Prof. D. Suryanarayana

Received: 19 December 2019 Revised: 21 January $2020 \quad$ Accepted: 5 March 2020

\begin{abstract}
A divisor $d$ of a positive integer $n$ is called a unitary divisor if $\operatorname{gcd}(d, n / d)=1$; and $d$ is called a bi-unitary divisor of $n$ if the greatest common unitary divisor of $d$ and $n / d$ is unity. The concept of a bi-unitary divisor is due to D. Surynarayana (1972). Let $\sigma^{* *}(n)$ denote the sum of the bi-unitary divisors of $n$. A positive integer $n$ is called a bi-unitary multiperfect number if $\sigma^{* *}(n)=k n$ for some $k \geq 3$. For $k=3$ we obtain the bi-unitary triperfect numbers.

Peter Hagis (1987) proved that there are no odd bi-unitary multiperfect numbers. The present paper is part III in a series of papers on even bi-unitary multiperfect numbers. In parts I and II we found all bi-unitary triperfect numbers of the form $n=2^{a} u$, where $1 \leq a \leq 5$ and $u$ is odd. There exist exactly six such numbers. In this part we examine the case $a=6$. We prove that if $n=2^{6} u$ is a bi-unitary triperfect number, then $n=22848, n=342720, n=51979200$ or $n=779688000$.
\end{abstract}

Keywords: Perfect numbers, Triperfect numbers, Multiperfect numbers, Bi-unitary analogues. 2010 Mathematics Subject Classification: 11A25.

\section{Introduction}

Throughout this paper, all lower case letters denote positive integers; $p$ and $q$ denote primes. The letters $u, v$ and $w$ are reserved for odd numbers. 
A divisor $d$ of $n$ is called a unitary divisor if $\operatorname{gcd}(d, n / d)=1$. If $d$ is a unitary divisor of $n$, we write $d \| n$. A divisor $d$ of $n$ is called a bi-unitary divisor if $(d, n / d)^{* *}=1$, where the symbol $(a, b)^{* *}$ denotes the greatest common unitary divisor of $a$ and $b$. The concept of a bi-unitary divisor is due to D. Suryanarayana (cf. [5]). Let $\sigma^{* *}(n)$ denote the sum of bi-unitary divisors of $n$. The function $\sigma^{* *}(n)$ is multiplicative, that is, $\sigma^{* *}(1)=1$ and $\sigma^{* *}(m n)=\sigma^{* *}(m) \sigma^{* *}(n)$ whenever $(m, n)=1$.

The concept of a bi-unitary perfect number was introduced by C. R. Wall [6]; a positive integer $n$ is called a bi-unitary perfect number if $\sigma^{* *}(n)=2 n$. C. R. Wall [6] proved that there are only three bi-unitary perfect numbers, namely 6,60 and 90 . A positive integer $n$ is called a bi-unitary multiperfect number if $\sigma^{* *}(n)=k n$ for some $k \geq 3$. For $k=3$ we obtain the bi-unitary triperfect numbers.

Peter Hagis [1] proved that there are no odd bi-unitary multiperfect numbers. Our present paper is part III in a series of papers on even bi-unitary multiperfect numbers. In part I (see [2]) we found all bi-unitary triperfect numbers of the form $n=2^{a} u$, where $1 \leq a \leq 3$ and $u$ is odd. We proved that if $1 \leq a \leq 3$ and $n=2^{a} u$ is a bi-unitary triperfect number, then $a=3$ and $n=120=2^{3}$.3.5. In part II (see [3]) we considered the cases $a=4$ and $a=5$. We proved that if $n=2^{4} u$ is a bi-unitary triperfect number, then $n=2160=2^{4} .3^{3} .5$, and that if $n=2^{5} u$ is a bi-unitary triperfect number, then $n=672=2^{5} .3 .7, n=10080=2^{5} .3^{2} .5 .7$, $n=528800=2^{5} \cdot 3.5^{2} .13$ or $n=22932000=2^{5} \cdot 3^{2} \cdot 5^{3} \cdot 7^{2} \cdot 13$.

In the present part we investigate bi-unitary triperfect numbers of the form $n=2^{6} u$. We prove in Theorem 3.1 that if $n=2^{6} u$ is a bi-unitary triperfect number, then $n=22848=$ $2^{6} .3 .7 .17, n=342720=2^{6} \cdot 3^{2} \cdot 5 \cdot 7.17, n=51979200=2^{6} .3 .5^{2} \cdot 7^{2} \cdot 13.17$ or $n=779688000=$ $2^{6} .3^{2} \cdot 5^{3} \cdot 7^{2} \cdot 13.17$.

To sum up, the cases $a=1$ and $a=2$ give no bi-unitary triperfect numbers, the cases $a=3$ and $a=4$ produce both one bi-unitary triperfect number, and the cases $a=5$ and $a=6$ yield both four bi-unitary triperfect numbers.

For a general account on various perfect-type numbers, we refer to [4].

\section{Preliminaries}

We assume that the reader has part I (see [2]) available. We, however, recall Lemmas 2.1 and 2.2 from part I, because they are so important also here.

Lemma 2.1. (I) If $\alpha$ is odd, then

$$
\frac{\sigma^{* *}\left(p^{\alpha}\right)}{p^{\alpha}}>\frac{\sigma^{* *}\left(p^{\alpha+1}\right)}{p^{\alpha+1}}
$$

for any prime $p$.

(II) For any $\alpha \geq 2 \ell-1$ and any prime $p$,

$$
\frac{\sigma^{* *}\left(p^{\alpha}\right)}{p^{\alpha}} \geq\left(\frac{1}{p-1}\right)\left(p-\frac{1}{p^{2 \ell}}\right)-\frac{1}{p^{\ell}}=\frac{1}{p^{2 \ell}}\left(\frac{p^{2 \ell+1}-1}{p-1}-p^{\ell}\right) .
$$

(III) If $p$ is any prime and $\alpha$ is a positive integer, then

$$
\frac{\sigma^{* *}\left(p^{\alpha}\right)}{p^{\alpha}}<\frac{p}{p-1} .
$$


Remark 2.1. (I) and (III) of Lemma 2.1 are mentioned in C. R. Wall [6]; (II) of Lemma 2.1 has been used by him [6] without explicitly stating it.

Lemma 2.2. Let $a>1$ be an integer not divisible by an odd prime $p$ and let $\alpha$ be a positive integer. Let $r$ denote the least positive integer such that $a^{r} \equiv 1\left(\bmod p^{\alpha}\right) ;$ then $r$ is usually denoted by $\operatorname{ord}_{p^{\alpha}}$ a. We have the following properties.

(i) If $r$ is even, then $s=r / 2$ is the least positive integer such that $a^{s} \equiv-1\left(\bmod p^{\alpha}\right)$. Also, $a^{t} \equiv-1\left(\bmod p^{\alpha}\right)$ for a positive integer $t$ if and only if $t=s u$, where $u$ is odd.

(ii) If $r$ is odd, then $p^{\alpha} \nmid a^{t}+1$ for any positive integer $t$.

Remark 2.2. Let $a, p, r$ and $s=r / 2$ be as in Lemma $2.2(\alpha=1)$. Then $p \mid a^{t}-1$ if and only if $r \mid t$. If $t$ is odd and $r$ is even, then $r \nmid t$. Hence $p \nmid a^{t}-1$. Also, $p \mid a^{t}+1$ if and only if $t=s u$, where $u$ is odd. In particular if $t$ is even and $s$ is odd, then $p \nmid a^{t}+1$. In order to check the divisibility of $a^{t}-1$ (when $t$ is odd) by an odd prime $p$, we can confine to those $p$ for which $\operatorname{ord}_{p} a$ is odd. Similarly, for examining the divisibility of $a^{t}+1$ by $p$ when $t$ is even, we need to consider primes $p$ with $s=\operatorname{ord}_{p} a / 2$ even.

\section{Bi-unitary triperfect numbers of the form $n=2^{6} u$}

Theorem 3.1. Assume that $n$ is a bi-unitary triperfect number with $2^{6} \| n$.

(a) Then $n=2^{6} \cdot 7^{b} \cdot 17^{c} . v$, where $b=1$ or $b=2$ and $v$ is prime to 2.7.17.

(b) If $b=1$, then $n=2^{6} \cdot 3 \cdot 7 \cdot 17=22848$ or $n=2^{6} \cdot 3^{2} \cdot 5 \cdot 7 \cdot 17=342720$.

(c) If $b=2$, then $n=2^{6} \cdot 3 \cdot 5^{2} \cdot 7^{2} \cdot 13 \cdot 17=51979200$ or $n=2^{6} \cdot 3^{2} \cdot 5^{3} \cdot 7^{2} \cdot 13 \cdot 17=779688000$.

Proof. Let $n=2^{6} u$, where $u$ is odd, be a bi-unitary triperfect number so that $\sigma^{* *}(n)=3 n$. Hence

$$
3.2^{6} \cdot u=3 n=\sigma^{* *}(n)=\sigma^{* *}\left(2^{6}\right) \sigma^{* *}(u)=7 \cdot 17 . \sigma^{* *}(u),
$$

so that

$$
3.2^{6} \cdot u=7.17 . \sigma^{* *}(u) .
$$

From (3.1), 7 and 17 are factors of $u$. So we may assume that $u=7^{b} .17^{c} . v$, where $v$ is odd and relatively prime to 7.17 . We now have

$$
n=2^{6} \cdot 7^{b} \cdot 17^{c} \cdot v
$$

Also, from (3.1),

$$
3.2^{6} \cdot 7^{b} \cdot 17^{c} \cdot v=7 \cdot 17 . \sigma^{* *}\left(7^{b}\right) \sigma^{* *}\left(17^{c}\right) \sigma^{* *}(v),
$$

and after simplification we get

$$
3.2^{6} \cdot 7^{b-1} \cdot 17^{c-1} \cdot v=\sigma^{* *}\left(7^{b}\right) \sigma^{* *}\left(17^{c}\right) \sigma^{* *}(v),
$$

where $v$ cannot have more than four odd prime factors. 
We prove Theorem 3.1 in this sequence: $(b),(c)$, and $(a)$.

Proof of $(b)$ of Theorem 3.1. Let $b=1$. Then taking $b=1$ in $(3.1 a)$ we obtain

$$
n=2^{6} \cdot 7 \cdot 17^{c} \cdot v \text {. }
$$

Since $\sigma^{* *}(7)=8$, taking $b=1$ in $(3.1 b)$, we get $3.2^{6} \cdot 17^{c-1} \cdot v=8 \cdot \sigma^{* *}\left(17^{c}\right) \sigma^{* *}(v)$ and on simplification we obtain

$$
3.2^{3} \cdot 17^{c-1} \cdot v=\sigma^{* *}\left(17^{c}\right) \sigma^{* *}(v)
$$

and $v$ has no more than two odd prime factors.

Case $(b=1, c=1)$. Let $c=1$. From $(3.2 b)$, we get $3 \cdot 2^{3} \cdot v=18 \cdot \sigma^{* *}(v)$ or

$$
2^{2} \cdot v=3 \cdot \sigma^{* *}(v) .
$$

This implies $3 \mid v$ so that $v=3^{d} . w$, where $(w, 2.3 .7 .17)=1$. From $(3.2 a)$ and $(3.2 c)$ we obtain

$$
n=2^{6} \cdot 7 \cdot 17.3^{d} \cdot w
$$

and

$$
2^{2} \cdot 3^{d-1} \cdot w=\sigma^{* *}\left(3^{d}\right) \cdot \sigma^{* *}(w),
$$

where $w$ has at most one odd prime factor.

We have for $d \geq 3, \frac{\sigma^{* *}\left(3^{d}\right)}{3^{d}} \geq \frac{112}{81}$. Hence for $d \geq 3$, from $(3.3 a)$, we obtain

$$
3=\frac{\sigma^{* *}(n)}{n} \geq \frac{119}{64} \cdot \frac{8}{7} \cdot \frac{18}{17} \cdot \frac{112}{81}=3.11>3
$$

a contradiction.

Hence $d=1$ or $d=2$.

Let $d=1$. From $(3.3 b), 2^{2} . w=4 . \sigma^{* *}(w)$, so that $w=\sigma^{* *}(w)$. Hence $w=1$. Thus $(3.3 b)$ is satisfied when $d=1$. So from $(3.3 a)(d=1), n=2^{6} .7 .17 .3=22848$ is a bi-unitary triperfect number.

Let $d=2$. From $(3.3 b),(d=2)$, we obtain $2^{2} \cdot 3 . w=10 . \sigma^{* *}(w)$ or

$$
\text { 2.3. } w=5 . \sigma^{* *}(w) .
$$

Hence $5 \mid w$. From (3.4), $w$ can have at most one odd prime factor and so $w=5^{e}$. Using this in (3.3a) and (3.4), we get

$$
n=2^{6} \cdot 7 \cdot 17 \cdot 3^{2} \cdot 5^{e}
$$

and

$$
2.3 .5^{e-1}=\sigma^{* *}\left(5^{e}\right)
$$

If $e \geq 2$, from $(3.4 b)$ it follows that $5 \mid \sigma^{* *}\left(5^{e}\right)$. This is not possible. Hence $e=1$ and for this value $(3.4 b)$ is satisfied. Thus $n=2^{6} .7 .17 .3^{2} .5=342720$ is a bi-unitary triperfect number.

The case $(b=1, c=1)$ is complete.

Case $(b=1, c \geq 2)$. The relevant equations are $(3.2 a)$ and $(3.2 b)$ with $c \geq 2$. We now prove that $n$ in $(3.2 a)$ cannot be a bi-unitary triperfect number when $c \geq 2$.

We obtain a contradiction to $(3.2 b)$, by examining the factors of $\sigma^{* *}\left(17^{c}\right)$. We distinguish the following cases: 
Case I. Let $c$ be odd so that $c \geq 3$. We have $\sigma^{* *}\left(17^{c}\right)=\frac{17^{c+1}-1}{16}$. Since $c+1$ is even, $17^{c+1} \equiv 1(\bmod 9)$. Hence $9 \mid \sigma^{* *}\left(17^{c}\right)$. From $(3.2 b)$, it follows that $3 \mid v$. Hence $v=3^{d} w$, where $w$ is prime to 2.3 .7 .17 ; using this in $(3.2 a)$ and (3.2b), we obtain

$$
n=2^{6} \cdot 7 \cdot 17^{c} \cdot 3^{d} \cdot w
$$

and

$$
2^{3} \cdot 17^{c-1} \cdot 3^{d+1} \cdot w=\sigma^{* *}\left(17^{c}\right) \cdot \sigma^{* *}\left(3^{d}\right) \cdot \sigma^{* *}(w),
$$

where $w$ has at most one odd prime factor.

Since $c \geq 3$, by Lemma $2.1(\ell=2), \frac{\sigma^{* *}\left(17^{c}\right)}{17^{c}} \geq \frac{88452}{83521}$; also, for $d \geq 3, \frac{\sigma^{* *}\left(3^{d}\right)}{3^{d}} \geq \frac{112}{81}$; using these results from $(3.5 a)$, we obtain for $d \geq 3$,

$$
3=\frac{\sigma^{* *}(n)}{n} \geq \frac{119}{64} \cdot \frac{8}{7} \cdot \frac{88452}{83521} \cdot \frac{112}{81}=3.11>3,
$$

a contradiction.

Hence $d=1$ or $d=2$.

If $d=1$, from $(3.5 a)$, we have

$$
3=\frac{\sigma^{* *}(n)}{n} \geq \frac{119}{64} \cdot \frac{8}{7} \cdot \frac{88452}{83521} \cdot \frac{4}{3}=3.000610625>3
$$

a contradiction.

Let $d=2$. From $(3.5 a)$ and $(3.5 b)$, we have $n=2^{6} \cdot 7 \cdot 17^{c} \cdot 3^{2} . w$, and

$$
2^{3} \cdot 17^{c-1} \cdot 3^{3} \cdot w=10 \cdot \sigma^{* *}\left(17^{c}\right) \cdot \sigma^{* *}(w) \text { or } 2^{2} \cdot 17^{c-1} \cdot 3^{3} \cdot w=5 \cdot \sigma^{* *}\left(17^{c}\right) \cdot \sigma^{* *}(w) ;
$$

the last equation implies that $5 \mid w$ and so $w=5^{e} . w^{\prime}$. Using this, we get

$$
n=2^{6} \cdot 7 \cdot 17^{c} \cdot 3^{2} \cdot 5^{e} \cdot w^{\prime}
$$

and

$$
2^{2} \cdot 17^{c-1} \cdot 3^{3} \cdot 5^{e-1} \cdot w^{\prime}=\sigma^{* *}\left(17^{c}\right) \cdot \sigma^{* *}\left(5^{e}\right) \cdot \sigma^{* *}\left(w^{\prime}\right) .
$$

From $(3.6 b)$, we have $w^{\prime}=1$. Rewriting $(3.6 a)$ and $(3.6 b)$, by replacing $w^{\prime}$ by 1 we get

$$
n=2^{6} \cdot 7 \cdot 17^{c} \cdot 3^{2} \cdot 5^{e},
$$

and

$$
2^{2} \cdot 17^{c-1} \cdot 3^{3} \cdot 5^{e-1} \cdot w^{\prime}=\sigma^{* *}\left(17^{c}\right) \cdot \sigma^{* *}\left(5^{e}\right)
$$

By Lemma 2.1, for $e \geq 3, \frac{\sigma^{* *}\left(5^{e}\right)}{5^{e}} \geq \frac{756}{625}$. Hence for $e \geq 3$, from $(3.6 a)$, we have

$$
3=\frac{\sigma^{* *}(n)}{n} \geq \frac{119}{64} \cdot \frac{8}{7} \cdot \frac{88452}{83521} \cdot \frac{10}{9} \cdot \frac{756}{625}=3.02461551>3
$$

a contradiction. 
Hence $e=1$ or $e=2$.

If $e=1$ then from $(3.6 a)^{\prime}$,

$$
3=\frac{\sigma^{* *}(n)}{n} \geq \frac{119}{64} \cdot \frac{8}{7} \cdot \frac{88452}{83521} \cdot \frac{10}{9} \cdot \frac{6}{5}=3.000610625>3,
$$

a contradiction.

Let $e=2$. From $(3.6 b)^{\prime}$, we have $2^{2} \cdot 17^{c-1} \cdot 3^{3} \cdot 5 \cdot w^{\prime}=26 \cdot \sigma^{* *}\left(17^{c}\right)$ or

$$
2.17^{c-1} \cdot 3^{3} \cdot 5 \cdot w^{\prime}=13 \cdot \sigma^{* *}\left(17^{c}\right)
$$

From the equation (3.6c), we infer that $w^{\prime}=1$. From (3.6c), we find that 13 divides its left-hand side. This is not possible. Hence $d=2$ is not possible.

Thus $n=2^{6} \cdot 7 \cdot 17^{c} . v$ cannot be a bi-unitary triperfect number when $c$ is odd and $c \geq 2$.

This completes Case I.

Case II. Let $c$ be even, so that $c=2 k$. Then

$$
\sigma^{* *}\left(17^{c}\right)=\left(\frac{17^{k}-1}{16}\right) \cdot\left(17^{k+1}+1\right)
$$

(i) Let $k$ be even. Then $32\left|17^{2}-1\right| 17^{k}-1$. Hence each of the factors on the right of $(3.7)$ is even so that $4 \mid \sigma^{* *}\left(17^{c}\right)$. From $(3.2 b)$ it follows that $v$ in $(3.2 b)$ can have at most one odd prime factor. Since $k$ is even, $9 \mid 17^{k}-1$ so that $9\left|\frac{17^{k}-1}{16}\right| \sigma^{* *}\left(17^{c}\right)$. Hence from $(3.2 b), 3 \mid v$ and so $v=3^{d}$. From $(3.2 a)$, we have

$$
n=2^{6} \cdot 7 \cdot 17^{c} \cdot 3^{d}
$$

Since $c=2 k$ and $k$ is even, $c \geq 4$. From $(3.7 a)$, for $d \geq 3$,

$$
3=\frac{\sigma^{* *}(n)}{n} \geq \frac{119}{64} \cdot \frac{8}{7} \cdot \frac{88452}{83521} \cdot \frac{112}{81}=3.111744352>3,
$$

a contradiction.

Hence $d=1$ or $d=2$.

Let $d=1$. From $(3.7 a),(d=1)$, we have

$$
3=\frac{\sigma^{* *}(n)}{n} \geq \frac{119}{64} \cdot \frac{8}{7} \cdot \frac{88452}{83521} \cdot \frac{4}{3}=3.0006106256>3,
$$

a contradiction.

Let $d=2$. From $(3.7 a),(d=2)$,

$$
3=\frac{\sigma^{* *}(n)}{n}<\frac{119}{64} \cdot \frac{8}{7} \cdot \frac{17}{16} \cdot \frac{10}{9}=2.508680556<3,
$$

a contradiction.

Hence $c=2 k$ and $k$ is even (or same as $4 \mid c$ ) is not admissible. 
(ii) Let $k$ be odd. We now prove that $k \geq 3$.

On the contrary, let $k=1$ so that $c=2$. Since $\sigma^{* *}\left(17^{2}\right)=290$, taking $c=2$ in $(3.2 b)$, we obtain after simplification,

$$
2^{2} \cdot 3.17 . v=5.29 \cdot \sigma^{* *}(v)
$$

It follows from (3.7b) that $v$ is divisible by 5 and 29. Since $v$ can have at most two odd prime factors, $v=5^{e} .29^{f}$. From $(3.2 a)$, we have $n=2^{6} .7 .17^{2} .5^{e} .29^{f}$, so that

$$
3=\frac{\sigma^{* *}(n)}{n}<\frac{119}{64} \cdot \frac{8}{7} \cdot \frac{290}{289} \cdot \frac{5}{4} \cdot \frac{29}{28}=2.760635504<3
$$

a contradiction.

Hence we may assume that $k \geq 3$. Hence $\frac{17^{k}-1}{16}>1$.

Since $k$ is odd, $16 \| 17^{k}-1$. Also, $2 \| 17^{k+1}+1$. Further, 3 neither divides $17^{k}-1$ nor $17^{k+1}+1$. Hence $\frac{17^{k}-1}{16}$ and $17^{k+1}+1$ are relatively prime. Also, $5 \mid 17^{t}-1$ if and only if $4 \mid t$. In particular, $t$ should be even. Since $k$ is odd, $5 \nmid 17^{k}-1$. If $p$ and $q$ are odd prime factors of $\frac{17^{k}-1}{16}$ and $17^{k+1}+1$, respectively, then $p \neq q, p \notin\{3,5,17\}$ and $q \notin\{3,17\}$.

If $\frac{k+1}{2}$ is odd, then $290=17^{2}+1 \mid 17^{k+1}+1$. In this case it follows from $(3.7)$ that $\sigma^{* *}\left(17^{c}\right)$ is divisible by three odd prime factors, namely, $p, 5$ and 29. From (3.2b), it follows that $v$ is divisible by these three odd prime factors; this leads to a contradiction since $v$ cannot have more than two odd prime factors.

If $\frac{k+1}{2}$ is even, then $4 \mid k+1$. And so, $5 \mid 17^{k+1}-1$. Hence $5 \nmid 17^{k+1}+1$. In this case $\sigma^{* *}\left(17^{c}\right)$ is divisible by two distinct odd primes $p$ and $q$; also, $p, q \notin\{3,5,17\}$. From (6b) it follows that $v$ is divisible by $p$ and $q$. Since $v$ has at most two odd prime factors, $v=p^{d} q^{e}$. Since $7 \nmid v$, we can assume that $p \geq 11$ and $q \geq 13$. From $(3.2 a), n=2^{6} \cdot 7 \cdot 17^{c} \cdot p^{d} . q^{e}$. Hence

$$
3=\frac{\sigma^{* *}(n)}{n}<\frac{119}{64} \cdot \frac{8}{7} \cdot \frac{17}{16} \cdot \frac{11}{10} \cdot \frac{13}{12}=2.690559896<3
$$

a contradiction.

The proof of Case II is complete.

The case $b=1$ is finished. This completes the proof of (b) of Theorem 3.1.

Proof of (c) of Theorem 3.1. Let $b=2$. Since $\sigma^{* *}\left(7^{2}\right)=50$, taking $b=2$ in $(3.1 b)$, we get after simplification, $3 \cdot 2^{5} \cdot 7 \cdot 17^{c-1} \cdot v=5^{2} \cdot \sigma^{* *}\left(17^{c}\right) \cdot \sigma^{* *}(v)$; this implies that $5^{2} \mid v$. Writing $v=5^{d} \cdot w$, where $d \geq 2$, we obtain from $(3.1 a)$ and $(3.1 b)$,

$$
n=2^{6} \cdot 7^{2} \cdot 17^{c} \cdot 5^{d} \cdot w, \quad(d \geq 2)
$$

and

$$
3 \cdot 2^{5} \cdot 7 \cdot 17^{c-1} \cdot 5^{d-2} \cdot w=\sigma^{* *}\left(17^{c}\right) \cdot \sigma^{* *}\left(5^{d}\right) \sigma^{* *}(w),
$$

and $w$ has no more than three odd prime factors and prime to 2.5.7.17. 
Case $(b=2, d=2)$. Since $\sigma^{* *}\left(5^{2}\right)=26$, from $(3.2 b)(d=2)$, we get after simplification,

$$
3 \cdot 2^{4} \cdot 7 \cdot 17^{c-1} \cdot w=13 \cdot \sigma^{* *}\left(17^{c}\right) \cdot \sigma^{* *}(w) .
$$

From this equation, we infer that $13 \mid w$. Let $w=13^{e} . w^{\prime}$. From $(3.8 a)$, we get,

$$
n=2^{6} \cdot 7^{2} \cdot 17^{c} \cdot 5^{2} \cdot 13^{e} \cdot w^{\prime}
$$

and from $(3.8 c)$, we have

$$
3 \cdot 2^{4} \cdot 7 \cdot 17^{c-1} \cdot 13^{e-1} \cdot w^{\prime}=\sigma^{* *}\left(17^{c}\right) \cdot \sigma^{* *}\left(13^{e}\right) \cdot \sigma^{* *}\left(w^{\prime}\right),
$$

where $w^{\prime}$ has at most two odd prime factors.

Let $c=1$. From (3.9b), $(c=1)$, we get after simplification

$$
2^{3} \cdot 7 \cdot 13^{e-1} \cdot w^{\prime}=3 \cdot \sigma^{* *}\left(13^{e}\right) \cdot \sigma^{* *}\left(w^{\prime}\right) .
$$

It follows from $(3.9 c)$ that $3 \mid w^{\prime}$. Let $w^{\prime}=3^{f} . w^{\prime \prime}$. From $(3.9 a)$, we have

$$
n=2^{6} \cdot 7^{2} \cdot 17 \cdot 5^{2} \cdot 13^{e} \cdot 3^{f} \cdot w^{\prime \prime}
$$

and from $(3.9 c)$,

$$
2^{3} \cdot 7 \cdot 13^{e-1} \cdot 3^{f-1} \cdot w^{\prime \prime}=\sigma^{* *}\left(13^{e}\right) \cdot \sigma^{* *}\left(3^{f}\right) \cdot \sigma^{* *}\left(w^{\prime \prime}\right),
$$

where $w^{\prime \prime}$ has at most one odd prime factor and prime to 2.3.5.7.13.17.

Let $e=1$ (already $b=2, d=2, c=1$ ). Taking $e=1$ in $(3.10 b)$, we get after simplification,

$$
2^{2} \cdot 3^{f-1} \cdot w^{\prime \prime}=\sigma^{* *}\left(3^{f}\right) \cdot \sigma^{* *}\left(w^{\prime \prime}\right)
$$

If $f=1$, then from $(3.10 c)$, we get $w^{\prime \prime}=\sigma^{* *}\left(w^{\prime \prime}\right)$ so that $w^{\prime \prime}=1$. Thus $(3.10 c)$ is satisfied when $f=1$. Taking $e=1, f=1$ and $w^{\prime \prime}=1$ in $(3.10 a)$, we see that $n=2^{6} \cdot 7^{2} \cdot 17.5^{2} \cdot 13.3=$ 51979200 is a bi-unitary triperfect number.

If $f=2$, from $(3.10 c)$, we find that $5 \mid w^{\prime \prime}$. But $w^{\prime \prime}$ is prime to 5 . So we may assume that $f \geq 3$; hence $\frac{\sigma^{* *}\left(3^{f}\right)}{3^{f}} \geq \frac{112}{81}$. From $(3.10 c)$, we have

$$
\frac{4}{3}=\frac{\sigma^{* *}\left(3^{f}\right)}{3^{f}} \cdot \frac{\sigma^{* *}\left(w^{\prime \prime}\right)}{w^{\prime \prime}} \geq \frac{\sigma^{* *}\left(3^{f}\right)}{3^{f}} \geq \frac{112}{81},
$$

which is false.

Hence $e=1$ is complete. Let $e=2$. From (3.10b), $(e=2)$, we get

$$
2^{3} \cdot 7 \cdot 13 \cdot 3^{f-1} \cdot w^{\prime \prime}=170 \cdot \sigma^{* *}\left(3^{f}\right) \cdot \sigma^{* *}\left(w^{\prime \prime}\right) .
$$

From $(3.10 d)$ it is clear that $5 \mid w^{\prime \prime}$ But this is false. have

We may assume that $e \geq 3$; so we can use $\frac{\sigma^{* *}\left(13^{e}\right)}{13^{e}} \geq \frac{30772}{28561}$. From $(3.10 a)$, for $f \geq 3$, we

$$
3=\frac{\sigma^{* *}(n)}{n} \geq \frac{119}{64} \cdot \frac{50}{49} \cdot \frac{18}{17} \cdot \frac{26}{25} \cdot \frac{30772}{28561} \cdot \frac{112}{81}=3.112527184>3,
$$

a contradiction.

Hence when $e \geq 3$, then $f=1$ or $f=2$. 
If $f=1$, from $(3.10 a)(f=1)$, we have

$$
3=\frac{\sigma^{* *}(n)}{n} \geq \frac{119}{64} \cdot \frac{50}{49} \cdot \frac{18}{17} \cdot \frac{26}{25} \cdot \frac{30772}{28561} \cdot \frac{4}{3}=3.001365498>3
$$

a contradiction.

If $f=2$, from $(3.10 b), 5 \mid w^{\prime \prime}$ which is false.

This proves that when $b=2$ and $d=2, c=1$ is not possible.

We continue assuming $b=2, d=2$ and let $c \geq 2$. The relevant equations are $(3.9 a)$ and $(3.9 b)$.

If $c=2$, since $\sigma^{* *}\left(17^{2}\right)=290$, from (3.9b), we find that $5 \mid w^{\prime}$ which is false. So, without loss of generality, we may assume that $c \geq 3$. Also, if $e=2$, since $\sigma^{* *}\left(13^{2}\right)=170$, from $(3.9 b)$, again we see that $5 \mid w^{\prime}$ which is false. Hence we may assume that $e \neq 2$.

We now assume that $3 \mid n$. From $(3.9 a), 3 \mid w^{\prime}$. Let $w^{\prime}=3^{f} . w^{\prime \prime}$. So from $(3.9 a)$, we have

$$
n=2^{6} \cdot 7^{2} \cdot 17^{c} \cdot 5^{2} \cdot 13^{e} \cdot 3^{f} \cdot w^{\prime \prime}
$$

and from $(3.9 b)$, we obtain

$$
2^{4} \cdot 7 \cdot 17^{c-1} \cdot 13^{e-1} \cdot 3^{f+1} \cdot w^{\prime \prime}=\sigma^{* *}\left(17^{c}\right) \cdot \sigma^{* *}\left(13^{e}\right) \cdot \sigma^{* *}\left(3^{f}\right) \cdot \sigma^{* *}\left(w^{\prime \prime}\right) ;
$$

$w^{\prime \prime}$ cannot have more than one odd prime factor.

If $f \geq 3$ and $e \geq 3$, from $(3.11 a)$, we have

$$
3=\frac{\sigma^{* *}(n)}{n} \geq \frac{119}{64} \cdot \frac{50}{49} \cdot \frac{88452}{83521} \cdot \frac{26}{25} \cdot \frac{30772}{28561} \cdot \frac{112}{81}=3.113160712>3,
$$

a contradiction.

Since $e \neq 2$, if $f \geq 3$, then the only possibility is $e=1$. Again from (3.11a), $(e=1)$, we have

$$
3=\frac{\sigma^{* *}(n)}{n} \geq \frac{119}{64} \cdot \frac{50}{49} \cdot \frac{88452}{83521} \cdot \frac{26}{25} \cdot \frac{14}{13} \cdot \frac{112}{81}=3.111744352>3,
$$

a contradiction.

Thus $f \geq 3$ does not hold. Hence $f=1$ or $f=2$.

Let $f=1$. If $e=1$, from $(3.11 a)$, we have

$$
3=\frac{\sigma^{* *}(n)}{n} \geq \frac{119}{64} \cdot \frac{50}{49} \cdot \frac{88452}{83521} \cdot \frac{26}{25} \cdot \frac{14}{13} \cdot \frac{4}{3}=3.000610625>3,
$$

a contradiction.

Since $e \neq 2$, we can assume $e \geq 3$. Again from (3.11a), we obtain

$$
3=\frac{\sigma^{* *}(n)}{n} \geq \frac{119}{64} \cdot \frac{50}{49} \cdot \frac{88452}{83521} \cdot \frac{26}{25} \cdot \frac{30772}{28561} \cdot \frac{4}{3}=3.001976401>3,
$$

a contradiction.

Hence $f=1$ cannot occur. If $f=2$, from $(3.11 b)$, we see that $5 \mid w^{\prime \prime}$ and this is false.

Thus the case $b=2, d=2$ when $3 \mid n$ is complete. 
Suppose that $3 \nmid n$ when $b=2, d=2$.

We return to the equations $(3.9 a)$ and $(3.9 b)$. In these two equations $w^{\prime}$ cannot have more than two odd prime factors. Hence we may assume that $w^{\prime}=p^{f} q^{g}$, where $p \geq 11$ and $q \geq 19$. Hence from $(3.9 a), n=2^{6} \cdot 7^{2} \cdot 17^{c} \cdot 5^{2} \cdot 13^{e} \cdot p^{f} \cdot q^{g}$ and so

a contradiction.

$$
3=\frac{\sigma^{* *}(n)}{n}<\frac{119}{64} \cdot \frac{50}{49} \cdot \frac{17}{16} \cdot \frac{26}{25} \cdot \frac{13}{12} \cdot \frac{11}{10} \cdot \frac{19}{18}=2.637175771<3
$$

Thus the case $b=2, d=2$ and $3 \nmid n$ is complete. This also finishes the case $b=2$ and $d=2$. Case ( $b=2, d \geq 3)$. We return to the equations $(3.8 a)$ and $(3.8 b)$, where we assume that $d \geq 3$. Case $(b=2, d=3)$. Taking $d=3$ in $(3.8 b)$ and since $\sigma^{* *}\left(5^{3}\right)=156=2^{2} .3 .13$, we get after simplification,

$$
2^{3} \cdot 7 \cdot 17^{c-1} \cdot 5 \cdot w=13 \cdot \sigma^{* *}\left(17^{c}\right) \cdot \sigma^{* *}(w) .
$$

From $(3.11 c), 13 \mid w$. Hence $w=13^{e} . w^{\prime}$ Substituting this in $(3.8 a)$ and $(3.11 c)$, we get

$$
n=2^{6} \cdot 7^{2} \cdot 17^{c} \cdot 5^{3} \cdot 13^{e} \cdot w^{\prime},
$$

and

$$
2^{3} \cdot 7 \cdot 17^{c-1} \cdot 5 \cdot 13^{e-1} \cdot w^{\prime}=\sigma^{* *}\left(17^{c}\right) \cdot \sigma^{* *}\left(13^{e}\right) \cdot \sigma^{* *}\left(w^{\prime}\right),
$$

where $w^{\prime}$ has at most one odd prime factor.

Let $c=1$ (already $b=2, d=3)$. Since $\sigma^{* *}(17)=18=2.3^{2}$, from $(3.12 b),(c=1)$, we get after simplification

$$
2^{2} \cdot 7 \cdot 5 \cdot 13^{e-1} \cdot w^{\prime}=3^{2} \cdot \sigma^{* *}\left(13^{e}\right) \cdot \sigma^{* *}\left(w^{\prime}\right) .
$$

From $(3.12 c), 3^{2} \mid w^{\prime}$ and so $w^{\prime}=3^{f}$, where $f \geq 2$. Hence from $(3.12 a)$ and $(3.12 c)$, we have

$$
n=2^{6} \cdot 7^{2} \cdot 17 \cdot 5^{3} \cdot 13^{e} \cdot 3^{f} \quad(f \geq 2),
$$

and

$$
2^{2} \cdot 7 \cdot 5 \cdot 13^{e-1} \cdot 3^{f-2}=\sigma^{* *}\left(13^{e}\right) \cdot \sigma^{* *}\left(3^{f}\right) .
$$

Let $e=1$. From $(3.13 b)(e=1)$, we get

$$
\text { 2.5.3 } 3^{f-2}=\sigma^{* *}\left(3^{f}\right) \text {. }
$$

If $f \geq 3$, from $(3.13 c), 3 \mid \sigma^{* *}\left(3^{f}\right)$, a contradiction. Hence $f=2$. It follows that $(3.13 c)$ is satisfied when $f=2$. Hence from (3.13a), $(e=1, f=2), n=2^{6} \cdot 7^{2} \cdot 17.5^{3} .13 .3^{2}=779688000$, is a bi-unitary triperfect number .

If $e=2$, since $\sigma^{* *}\left(13^{2}\right)=170$, from $(3.13 b), 17$ is a factor of the left-hand side of $(3.13 b)$. But this is not true.

We may assume that $e \geq 3$.

Let $f=2$. From (3.13b), $(f=2)$, we get after simplification, 2.7.13 $3^{e-1}=\sigma^{* *}\left(13^{e}\right)$; from this equation since $e \geq 3$, we see that $13 \mid \sigma^{* *}\left(13^{e}\right)$ which is false. Hence $f \geq 3$.

Thus $e$ and $f$ are both $\geq 3$. From (3.13a), we now have

a contradiction.

$$
3=\frac{\sigma^{* *}(n)}{n} \geq \frac{119}{64} \cdot \frac{50}{49} \cdot \frac{18}{17} \cdot \frac{156}{125} \cdot \frac{30772}{28561} \cdot \frac{112}{81}=3.73503262>3,
$$

Thus $c=1$ is not possible. 
Let $c \geq 2$ (with $b=2, d=3$ ). We return to the equations $(3.12 a)$ and $(3.12 b)$, where now $c \geq 2$. In $(3.12 a), w^{\prime}$ has at most one odd prime factor.

If $3 \nmid n$, then $w^{\prime}=1$ or $p^{f}$, where $p \geq 11$. In any case $\frac{\sigma^{* *}\left(w^{\prime}\right)}{w^{\prime}}<\frac{11}{10}$. Hence if $3 \nmid n$, from $(3.12 a)$, we have

$$
3=\frac{\sigma^{* *}(n)}{n}<\frac{119}{64} \cdot \frac{50}{49} \cdot \frac{17}{16} \cdot \frac{156}{125} \cdot \frac{13}{12} \cdot \frac{11}{10}=2.998052455<3,
$$

a contradiction.

Suppose that $3 \mid n$. Then $w^{\prime}=3^{f}$. From $(3.12 a)$ and $(3.12 b)$,

$$
n=2^{6} \cdot 7^{2} \cdot 17^{c} \cdot 5^{3} \cdot 13^{e} \cdot 3^{f},
$$

and

$$
2^{3} \cdot 7 \cdot 17^{c-1} \cdot 5 \cdot 13^{e-1} \cdot 3^{f}=\sigma^{* *}\left(17^{c}\right) \cdot \sigma^{* *}\left(13^{e}\right) \cdot \sigma^{* *}\left(3^{f}\right) .
$$

If $f \geq 3$, from $(3.12 c)$, we get

$$
3=\frac{\sigma^{* *}(n)}{n} \geq \frac{119}{64} \cdot \frac{50}{49} \cdot \frac{156}{125} \cdot \frac{112}{81}=3.274074074>3,
$$

a contradiction; in the above we used that $\frac{\sigma^{* *}\left(3^{f}\right)}{3^{f}} \geq \frac{112}{81}$ for $f \geq 3$; also, $\frac{\sigma^{* *}\left(17^{c}\right)}{17^{c}} \geq 1$ and $\frac{\sigma^{* *}\left(13^{e}\right)}{13^{e}} \geq 1$.

Hence $f=1$ or $f=2$.

If $f=1$, from $(3.12 d)$, it follows that its right-hand side is divisible by $2^{4}$, whereas its lefthand side is divisible unitarily by $2^{3}$.

Let $f=2$. Taking $f=2$ in $(3.12 c)$ and $(3.12 d)$, we obtain

$$
n=2^{6} \cdot 7^{2} \cdot 17^{c} \cdot 5^{3} \cdot 13^{e} \cdot 3^{2}
$$

and

$$
2^{2} \cdot 7 \cdot 17^{c-1} \cdot 13^{e-1} \cdot 3^{2}=\sigma^{* *}\left(17^{c}\right) \cdot \sigma^{* *}\left(13^{e}\right) .
$$

Since $\sigma^{* *}\left(17^{2}\right)=290$, taking $c=2$ in $(3.13 b)$, we see that the left-hand side of it should be divisible by 29 and this is not possible. Hence we may assume that $c \geq 3$; hence we can use the result $\frac{\sigma^{* *}\left(17^{c}\right)}{17^{c}} \geq \frac{88452}{83521}$.

If $e \geq 3$, then $\frac{\sigma^{* *}\left(13^{e}\right)}{13^{e}} \geq \frac{30772}{28561}$. Hence if $e \geq 3$, from $(3.13 a)$, we have

$$
3=\frac{\sigma^{* *}(n)}{n} \geq \frac{119}{64} \cdot \frac{50}{49} \cdot \frac{88452}{83521} \cdot \frac{156}{125} \cdot \frac{30772}{28561} \cdot \frac{10}{9}=3.001976401>3,
$$

a contradiction.

Hence $e=1$ or $e=2$.

If $e=1,(3.13 b)$ reduces to $2.7 .17^{c-1} .3^{2}=\sigma^{* *}\left(17^{c}\right)$; this implies that $17 \mid \sigma^{* *}\left(17^{c}\right)$ which is false.

If $e=2$, since $\sigma^{* *}\left(13^{2}\right)=170$, taking $e=2$ in $(3.13 b)$, we see that 5 should divide its left-hand side. But this is not possible.

The case $b=2$ and $d=3$ is complete. 
Case $(b=2, d \geq 4)$. The relevant equations are $(3.8 a)$ and $(3.8 b)$, where $d \geq 4$.

Case $(b=2, d \geq 4,3 \mid n)$. Since $3 \mid n$, we have $3 \mid w$. Let $w=3^{e} . w^{\prime}$. Using this in $(3.8 a)$ and $(3.8 b)$, we get

$$
n=2^{6} \cdot 7^{2} \cdot 17^{c} \cdot 5^{d} \cdot 3^{e} \cdot w^{\prime}, \quad(d \geq 4)
$$

and

$$
3 \cdot 2^{5} \cdot 7 \cdot 17^{c-1} \cdot 5^{d-2} \cdot 3^{e} \cdot w^{\prime}=\sigma^{* *}\left(17^{c}\right) \cdot \sigma^{* *}\left(5^{d}\right) \cdot \sigma^{* *}\left(3^{e}\right) \cdot \sigma^{* *}\left(w^{\prime}\right),
$$

and $w^{\prime}$ has no more than two odd prime factors and is prime to 2.3.5.7.17.

Since $d \geq 3$, we have $\frac{\sigma^{* *}\left(5^{d}\right)}{5^{d}} \geq \frac{756}{625}$ and for $e \geq 3, \frac{\sigma^{* *}\left(3^{e}\right)}{3^{e}} \geq \frac{112}{81}$. Hence for $e \geq 3$, from $(3.14 a)$,

$$
3=\frac{\sigma^{* *}(n)}{n} \geq \frac{119}{64} \cdot \frac{50}{49} \cdot \frac{756}{625} \cdot \frac{112}{81}=3.173>3,
$$

a contradiction.

Hence $e=1$ or $e=2$.

If $e=1$, again from $(3.14 a),(e=1)$, we have

$$
3=\frac{\sigma^{* *}(n)}{n} \geq \frac{119}{64} \cdot \frac{50}{49} \cdot \frac{756}{625} \cdot \frac{4}{3}=3.06>3
$$

a contradiction.

Let $e=2$ (with $b=2, d \geq 4$ ). Taking $e=2$ in $(3.14 a)$ and (3.14b), we get

$$
n=2^{6} \cdot 7^{2} \cdot 17^{c} \cdot 5^{d} \cdot 3^{2} \cdot w^{\prime}, \quad(d \geq 4)
$$

and

$$
2^{4} \cdot 7 \cdot 17^{c-1} \cdot 5^{d-3} \cdot 3^{3} \cdot w^{\prime}=\sigma^{* *}\left(17^{c}\right) \cdot \sigma^{* *}\left(5^{d}\right) \cdot \sigma^{* *}\left(w^{\prime}\right)
$$

and $w^{\prime}$ has no more than two odd prime factors and is prime to 2.3.5.7.17.

When $e=2$, we wish to show that $n$ (hence $w^{\prime}$ ) is not divisible by 11 or 13 or 19 or 23 . If this is proved, then if $w^{\prime}$ is divisible by two odd primes (in the worst case) say $p$ and $q$, then $w^{\prime}=p^{f} \cdot q^{g}$, where we can assume that $p \geq 29$ and $q \geq 31$. Also, from $(3.15 a), n=2^{6} \cdot 7^{2} \cdot 17^{c} \cdot 5^{d} \cdot 3^{2} \cdot p^{f} \cdot q^{g}$ so that

$$
3=\frac{\sigma^{* *}(n)}{n}<\frac{119}{64} \cdot \frac{50}{49} \cdot \frac{17}{16} \cdot \frac{5}{4} \cdot \frac{10}{9} \cdot \frac{29}{28} \cdot \frac{31}{30}=2.996523995<3,
$$

a contradiction. With this the case $b=2, d \geq 4,3 \mid n$ would be complete.

We now prove that $n$ in $(3.15 a)$ and (3.15b) is not divisible by $s$, where $s \in\{11,13,19,23\}$.

We assume that $s \mid n$ so that $s \mid w^{\prime}$. Let $w^{\prime}=s^{f} . w^{\prime \prime}$; substituting this into $(3.15 a)$ and $(3.15 b)$, we obtain

$$
n=2^{6} \cdot 7^{2} \cdot 17^{c} \cdot 5^{d} \cdot 3^{2} \cdot s^{f} \cdot w^{\prime \prime}, \quad(d \geq 4)
$$

and

$$
2^{4} \cdot 7 \cdot 17^{c-1} \cdot 5^{d-3} \cdot 3^{3} \cdot s^{f} \cdot w^{\prime \prime}=\sigma^{* *}\left(17^{c}\right) \cdot \sigma^{* *}\left(5^{d}\right) \cdot \sigma^{* *}\left(s^{f}\right) \cdot \sigma^{* *}\left(w^{\prime \prime}\right),
$$

and $w^{\prime}$ has no more than one odd prime factor and is prime to 2.3.5.7.17.s.

We now examine the factors of $\sigma^{* *}\left(5^{d}\right)$ in the presence of $(3.16 a)$ and $(3.16 b)$. We distinguish the following cases: 
Case A. Let $d$ be odd. Then

$$
\sigma^{* *}\left(5^{d}\right)=\frac{5^{d+1}-1}{4}=\frac{\left(5^{t}-1\right)\left(5^{t}+1\right)}{4}, \quad\left(t=\frac{d+1}{2}\right) .
$$

(i) Let $t$ be even. Then $8 \mid 5^{t}-1$ and trivially $2 \mid 5^{t}+1$. Hence $4 \mid \frac{\left(5^{t}-1\right)\left(5^{t}+1\right)}{4}=\sigma^{* *}\left(5^{d}\right)$; it follows from $(3.15 b)$ that $w^{\prime \prime}=1$. Rewriting $(3.16 a)$ and $(3.16 b)$, taking $w^{\prime \prime}=1$, we get

$$
n=2^{6} \cdot 7^{2} \cdot 17^{c} \cdot 5^{d} \cdot 3^{2} \cdot s^{f}, \quad(d \geq 4)
$$

and

$$
2^{4} \cdot 7 \cdot 17^{c-1} \cdot 5^{d-3} \cdot 3^{3} \cdot s^{f}=\sigma^{* *}\left(17^{c}\right) \cdot \sigma^{* *}\left(5^{d}\right) \cdot \sigma^{* *}\left(s^{f}\right) .
$$

If $s=19$ or 23 , so that $s \geq 19$, from (3.16c), we obtain

$$
3=\frac{\sigma^{* *}(n)}{n}<\frac{119}{64} \cdot \frac{50}{49} \cdot \frac{17}{16} \cdot \frac{5}{4} \cdot \frac{10}{9} \cdot \frac{19}{18}=2.955414841<3,
$$

a contradiction.

We may assume that $s=11$ or 13 . We have:

(a) $3 \mid 5^{t}-1$, since $t$ is even.

(b) $9\left|5^{t}-1 \Longleftrightarrow 6\right| t \Longleftrightarrow 7\left|5^{t}-1 ; 6\right| t$ implies $5^{6}-1 \mid 5^{t}-1$ and $5^{6}-1=2^{3} \cdot 3^{2} .7 .31$. Hence $31 \mid 5^{t}-1$ so that $31\left|\frac{5^{t}-1}{2}\right| \sigma^{* *}\left(5^{d}\right)$. This is not possible from $(3.16 d)$. Hence $9 \nmid 5^{t}-1$ and $7 \nmid 5^{t}-1$. As a consequence, $3 \| 5^{t}-1$.

(c) Since $t$ is even, $8 \mid 5^{t}-1$; but $16 \mid 5^{t}-1$ implies that $8 \mid \sigma^{* *}\left(5^{d}\right)$. This results in an imbalance in the powers of two between two sides of $(3.16 d)$. Hence $16 \nmid 5^{t}-1$ and so $8 \| 5^{t}-1$.

(d) $11\left|5^{t}-1 \Longleftrightarrow 5\right| t$; and $5 \mid t$ implies that $5^{5}-1 \mid 5^{t}-1$. Also, $5^{5}-1=2^{2} .11 .71$. Hence $71\left|\frac{5^{t}-1}{2}\right| \sigma^{* *}\left(5^{d}\right)$; this is not possible from $(3.16 d)$. Thus $11 \nmid 5^{t}-1$.

(e) $13\left|5^{t}-1 \Longleftrightarrow 4\right| t$; this implies $16\left|5^{4}-1\right| 5^{t}-1$. In (c) above, we proved that $16 \nmid 5^{t}-1$. Hence $13 \nmid 5^{t}-1$.

(f) $17\left|5^{t}-1 \Longleftrightarrow 16\right| t$; this implies $4 \mid t$. As in (e), we get a contradiction. Hence $17 \nmid 5^{t}-1$.

We have $d \geq 5$, since $d$ is odd and $d \geq 4$. Hence $t=\frac{d+1}{2} \geq 3$. It is clear that $\frac{5^{t}-1}{24}>1$, odd and not divisible by 3 . Hence $\frac{5^{t}-1}{24}$ must be divisible by an odd prime say $p$. Since $5^{t}-1$ is not divisible by any of the primes $5,7,11,13$ and 17 , the same is true with respect to $\frac{5^{t}-1}{24}$. Hence $p\left|\frac{5^{t}-1}{24}\right| \sigma^{* *}\left(5^{d}\right)$ and $p \notin\{2,3,5,7,11,13,17\}$. This contradicts $(3.16 d)$ since $s=11$ or 13 .

The case that $t$ is even is complete.

(ii) Let $t$ be odd.

(a) $4 \| 5^{t}-1$ since $t$ is odd. Hence $\frac{5^{t}-1}{4}$ is odd and $>1$ since $t \geq 3$.

(b) $5^{t}-1$ is not divisible by $3,7,13,17$ or 23 , since $t$ is odd; trivially not divisible by 5 . 
(c) $19\left|5^{t}-1 \Longleftrightarrow 9\right| t$; this implies that $5^{9}-1 \mid 5^{t}-1$. Also, $5^{9}-1=2^{2} .19 .31 .829$. Hence $\frac{5^{t}-1}{4} \mid \sigma^{* *}\left(5^{d}\right)$, is divisible by 31 and 829 . It follows from $(3.16 b)$ that $w^{\prime \prime}$ is divisible by 31 and 829 . This is not possible since $w^{\prime \prime}$ cannot have more than one odd prime factor. Hence $19 \nmid 5^{t}-1$.

(d) Let $s \neq 11$. We claim that $11 \nmid 5^{t}-1$. Suppose that $11 \mid 5^{t}-1$. This is if and only if $5 \mid t$. Hence $11 \mid 5^{t}-1$ implies $5^{5}-1 \mid 5^{t}-1$. Also, $5^{5}-1=2^{2} .11 .71$. It follows that $\frac{5^{t}-1}{4} \mid \sigma^{* *}\left(5^{d}\right)$, is divisible by 11 and 71 . Since $s \neq 11$, from $(3.16 b)$ we infer that $w^{\prime \prime}$ is divisible by 11 and 71 . This is not possible since $w^{\prime \prime}$ cannot have more than one odd prime factor. Hence when $s \neq 11,11 \nmid 5^{t}-1$.

(e) Let $s=11$. We prove that $\frac{5^{t}-1}{4}$ has a prime factor $\neq 11$; if $11 \nmid 5^{t}-1$, then this is trivially true. We assume that $11 \mid 5^{t}-1$. If $\frac{5^{t}-1}{4}$ is divisible by 11 alone, then we must have $\frac{5^{t}-1}{4}=11^{\alpha}$ for some positive integer $\alpha$. If $\alpha \geq 2$, then $11^{2} \mid 5^{t}-1$; this is if and only if $55 \mid t$. In particular $11 \mid t$. Hence $5^{11}-1 \mid 5^{t}-1$ and $5^{11}-1=2^{2} .12207031$. It follows that $12207031 \mid \frac{5^{t}-1}{4}=11^{\alpha}$, which is impossible. Hence $\frac{5^{t}-1}{4}=11$ or $5^{t}=45$, which is not possible. Thus $\frac{5^{t}-1}{4}$ must be divisible by an odd prime say $p \neq 11$. Clearly, $p \notin\{3,5,7,11,13,17,19,23\}$. Hence $p\left|\frac{5^{t}-1}{4}\right| \sigma^{* *}\left(5^{d}\right)$. From $(3.16 b)$, we find that $p \mid w^{\prime \prime}$.

Thus if $s \neq 11$, from (a)-(d), it follows that $\frac{5^{t}-1}{4}$ is not divisible by any prime in the set $\{3,5,7,11,13,17,19,23\}$. In particular, if $p\left|\frac{5^{t}-1}{4}\right| \sigma^{* *}\left(5^{d}\right)$ and $p \notin\{3,5,7,11,13,17,19,23\}$, from $(3.16 b)$, we infer that $p \mid w^{\prime \prime}$.

Hence when $t$ is odd, we can conclude that there is an odd prime $p \mid \frac{5^{t}-1}{4}$ and $p \mid w^{\prime \prime}$. Let $s \in\{11,13,19,23\}$. We now prove that we can find an odd prime $q \mid 5^{t}+1$ and $q \mid w^{\prime \prime}$ when $t$ is odd. We have

(f) $2 \| 5^{t}+1$ and $3 \mid 5^{t}+1$.

(g) $5^{t}+1$ is not divisible by 13 and 17 since $t$ is odd.

(h) $5^{t}+1$ is not divisible by 11 and 19 for any $t$.

(i) $23 \mid 5^{t}+1 \Longleftrightarrow t=11 u$, where $u$ is odd. Hence $23 \mid 5^{t}+1$ implies $5^{11}+1 \mid 5^{t}+1$. Also, $5^{11}+1=2.3 .23 .67 .5281$. So, $5^{t}+1$, a factor of $\sigma^{* *}\left(5^{d}\right)$, is divisible by 67 and 5281 . From $(3.16 b)$, it follows that $w^{\prime \prime}$ is divisible by 67 and 5281 . This cannot happen. Hence $23 \nmid 5^{t}+1$.

Thus $5^{t}+1$ is not divisible by any of $11,13,17,19$ and 23 .

(j) We may note that $7\left|5^{t}+1 \Longleftrightarrow 9\right| 5^{t}+1 \Longleftrightarrow t=3 u$, where $u$ is odd. Assume that $7 \nmid 5^{t}+1$. Then $9 \nmid 5^{t}+1$. Hence $3 \| 5^{t}+1$. Also, $\frac{5^{t}+1}{6}>1$, odd and not divisible by any of the primes $3,5,7,11,13,17,19$ and 23 . Let $q \mid \frac{5^{t}+1}{6}$ so that $q \mid \sigma^{* *}\left(5^{d}\right)$. Then $q \notin\{3,5,7,11,13,17,19,23\}$. From $(3.16 b), q \mid w^{\prime \prime}$.

Suppose that $7 \mid 5^{t}+1$ so that $9 \mid 5^{t}+1$. We note that $27 \mid 5^{t}+1 \Longleftrightarrow t=9 u$, where $u$ is odd. Hence $27 \mid 5^{t}+1$ implies $5^{9}+1 \mid 5^{t}+1$. Also, $5^{9}+1=2.3^{3} .7 .5167$. Hence 
$5167\left|5^{t}+1\right| \sigma^{* *}\left(5^{d}\right)$. From $(3.16 b)$, it follows that $5167 \mid w^{\prime \prime}$. We already proved that there is an odd prime $p \mid \frac{5^{t}-1}{4}$ and $p \mid w^{\prime \prime}$. Now, $\frac{5^{t}-1}{4}$ and $5^{t}+1$ are relatively prime. Since $p$ and 5167 respectively divide these factors, it follows that $w^{\prime \prime}$ is divisible by these two odd primes. This cannot happen. Hence $27 \nmid 5^{t}+1$. Thus $7 \mid 5^{t}+1$ implies $9 \| 5^{t}+1$.

We have $\frac{5^{t}+1}{18}$ is $>1$, odd and not divisible by 3. By our assumption, $7 \mid \frac{5^{t}+1}{18}$ and from $(3.16 b), 7^{2} \nmid \frac{5^{t}+1}{18}$, since $\frac{5^{t}+1}{18} \mid \sigma^{* *}\left(5^{d}\right)$. Hence $7 \| \frac{5^{t}+1}{18}$. If $\frac{5^{t}+1}{18}$ is divisible by 7 alone, then we must have $\frac{5^{t}+1}{18}=7$ or $5^{t}=125$ or $t=3$.

We now prove that $t=3$ is not possible. Suppose that $3=t=\frac{d+1}{2}$ so that $d=5$. We have $\sigma^{* *}\left(5^{5}\right)=\frac{5^{6}-1}{4}=2.3^{2} .7 .31$. Taking $d=5$ in (3.16b), we get after simplification

$$
2^{3} \cdot 3 \cdot 17^{c-1} \cdot 5^{2} \cdot s^{f} \cdot w^{\prime \prime}=31 \cdot \sigma^{* *}\left(17^{c}\right) \cdot \sigma^{* *}\left(s^{f}\right) \cdot \sigma^{* *}\left(w^{\prime \prime}\right) ;
$$

this implies that $31 \mid w^{\prime \prime}$ so that $w^{\prime \prime}=31^{g}$. Substituting $w^{\prime \prime}=31^{g}$ in $(3.16 a)$ and (3.16e), we get

$$
n=2^{6} \cdot 7^{2} \cdot 17^{c} \cdot 5^{d} \cdot 3^{2} \cdot s^{f} \cdot 31^{g},
$$

and

$$
2^{3} \cdot 3 \cdot 17^{c-1} \cdot 5^{2} \cdot s^{f} \cdot 31^{g-1}=\sigma^{* *}\left(17^{c}\right) \cdot \sigma^{* *}\left(s^{f}\right) \cdot \sigma^{* *}\left(31^{g}\right) .
$$

We obtain a contradiction by examining the factors of $\sigma^{* *}\left(17^{c}\right)$.

Let $c$ be odd. Then $9 \mid \sigma^{* *}\left(17^{c}\right)$. This is not possible from $(3.17 b)$.

We may assume that $c$ is even so that $c=2 k$. Then

$$
\sigma^{* *}\left(17^{c}\right)=\left(\frac{17^{k}-1}{16}\right) \cdot\left(17^{k+1}+1\right) .
$$

(i) If $k$ is even, $9 \mid 17^{k}-1$ and so $9\left|\frac{17^{k}-1}{16}\right| \sigma^{* *}\left(17^{c}\right)$ and this leads to a contradiction from $(3.17 b)$.

(ii) Let $k$ be odd. First we note that $k>1$. If $k=1$, then $c=2$. We have $\sigma^{* *}\left(17^{2}\right)=290$. Taking $c=2$ in $(3.17 b)$, we see that 29 divides its right-hand side but 29 does not divide its left-hand side. Hence $k=1$ cannot occur.

We may assume that $k \geq 3$. Since $k$ is odd, $16 \| 17^{k}-1$; also, $17^{k}-1$ is not divisible by $3,5,7,11,13,23$ and 31 since $k$ is odd. $19\left|17^{k}-1 \Longleftrightarrow 9\right| k$. In such a case $17^{9}-1 \mid 17^{k}-1$. Also, $17^{9}-1=2^{4} .19 .307 .1270657$. In particular, 307| $\frac{17^{k}-1}{16} \mid \sigma^{* *}\left(17^{c}\right)$. But this is not possible can be seen from $(3.17 b)$. Hence $19 \nmid 17^{k}-1$.

Thus $\frac{17^{k}-1}{16}>1$ and is odd; also it is not divisible by $3,5,7,11,13,17,19,23$ and 31 . If $p$ is an odd prime factor of $\frac{17^{k}-1}{16} \mid \sigma^{* *}\left(17^{c}\right)$, then $p \notin\{3,5,7,11,13,17,19,23,31\}$. But this is not possible from $(3.17 b)$.

Thus $d=5$ (or $t=3$ ) is not admissible. 
Hence $\frac{5^{t}+1}{18}$ is not divisible by 7 alone. As a consequence, we can find an odd prime $q \mid \frac{5^{t}+1}{18}$ and $q \neq 7$. Since $\frac{5^{t}+1}{18}$ is not divisible by $3,5,7,11,13,17,19$ and 23 , $q \notin\{3,5,7,11,13,17,19,23\}$. It follows from (3.16b) that $q \mid w^{\prime \prime}$. Since $p\left|w^{\prime \prime}, q\right| w^{\prime \prime}$ and $p \neq q$ it follows that $w^{\prime \prime}$ is divisible by two odd primes which is not possible.

This completes the case when $t=\frac{d+1}{2}$ is odd.

Case B. It remains to examine the case when $d$ is even. Let $d=2 k$. Then

$$
\sigma^{* *}\left(5^{d}\right)=\left(\frac{5^{k}-1}{4}\right) \cdot\left(5^{k+1}+1\right) \text {. }
$$

(iii) If $k$ is even, we get a contradiction just as in (i) of Case A where $t$ was even.

(iv) Assume that $k$ is odd. Since $d=2 k \geq 4$, we have $k \geq 3$. Again as in (ii) of Case A, on similar lines, we can show that $\frac{5^{k}-1}{4}$ is divisible by an odd prime $p \mid w^{\prime \prime}$.

It remains to examine $5^{k+1}+1$ when $k$ is odd.

(v) Since $k+1$ is even, $2 \| 5^{k+1}+1$ and not divisible by 3,7 and 23 .

(vi) Since $5^{t}+1$ is not divisible by 11 and 19 for any positive integer $t$; the same is true with respect to $5^{k+1}+1$.

(vii) $17 \mid 5^{k+1}+1 \Longleftrightarrow k+1=8 u$, where $u$ is odd. Hence $17 \mid 5^{k+1}+1$ implies $5^{8}+1 \mid 5^{k+1}+1$. Also, $5^{8}+1=2.17 .11489$. Hence $11489\left|5^{k+1}+1\right| \sigma^{* *}\left(5^{d}\right)$. From $(3.16 b), 11489 \mid w^{\prime \prime}$. Since $p \mid \frac{5^{k}-1}{4}$ divides $w^{\prime \prime}$, it follows that $w^{\prime \prime}$ is divisible by two odd primes. This cannot happen. Hence $17 \nmid 5^{k+1}+1$.

(viii) If $13 \nmid 5^{k+1}+1$, then it follows from (v)-(vi) that $\frac{5^{k+1}+1}{2}>1$, is odd and not divisible by any of the primes $3,5,7,11,13,19$ and 23 . Thus if $q \mid \frac{5^{k+1}+1}{2}$, then $q$ is odd and $q \notin\{3,5,7,11,13,19,23$. $\}$. From $(3.16 b), q \mid w^{\prime \prime}$.

Suppose that $13 \mid 5^{k+1}+1$. Suppose that $13^{2} \mid 5^{k+1}+1$; this is if and only if $k+1=26 u$, where $u$ is odd. This implies that $5^{26}+1 \mid 5^{k+1}+1$ and $5^{26}+1=$ $2.13^{2} .53 .83181652304609$. Thus $\frac{5^{k+1}+1}{2}$ is divisible by two odd primes and these primes divide $w^{\prime \prime}$ by $(3.16 b)$. But this is not possible. Hence $13 \| \frac{5^{k+1}+1}{2}$. It follows that $\frac{5^{k+1}+1}{26}>1$, odd and not divisible by any of $3,5,7,11,13,17,19$ and 23 . Hence if $q \mid \frac{5^{k+1}+1}{26}$, then $q$ is odd and $q \notin\{3,5,7,11,13,19,23\}$. From $(3.16 b), q \mid w^{\prime \prime}$.

Thus $p$ and $q$ divide $w^{\prime \prime}$. This is not possible.

This completes the Case B.

Hence $s \nmid n$, where $s \in\{11,13,19,23\}$.

Thus, $n$ in $(3.16 a)$ satisfying $(3.16 b)$ cannot be a bi-unitary triperfect number when $b=2$, $d \geq 4$ and $3 \mid n$. 
Case $(b=2, d \geq 4,3 \nmid n)$. The relevant equations are $(3.8 a)$ and $(3.8 b)$. We obtain a contradiction by examining the factors of $\sigma^{* *}\left(17^{c}\right)$, and hence $n$ in $(3.8 a)$ cannot be a bi-unitary triperfect number.

For the validity of (3.8b), we show that the only choice for $c$ is that $c=2 k$, where $k$ is odd. In such a case, we prove that $\frac{17^{k}-1}{16}$ and $17^{k+1}+1$ should be divisible by two odd primes $p$ and $q$, and each of them exceeds 41 . We can assume that $p \geq 43$ and $q \geq 47$. If at all $w$ has a third prime factor say $r$, then obviously $r \geq 11$, from $(3.8 b)$. Hence $n=2^{6} \cdot 7^{2} \cdot 17^{c} \cdot 5^{d} \cdot p^{e} \cdot q^{f} \cdot r^{g}$. We have

$$
3=\frac{\sigma^{* *}(n)}{n}<\frac{119}{64} \cdot \frac{50}{49} \cdot \frac{17}{16} \cdot \frac{5}{4} \cdot \frac{43}{42} \frac{47}{46} \cdot \frac{11}{10}=2.899557597<3,
$$

a contradiction.

If $c$ is odd or $4 \mid c$, then $9 \mid \sigma^{* *}\left(17^{c}\right)$. This implies that $3 \mid w$, from $(3.8 b)$. This is not true since by our assumption $3 \nmid n$.

Let $c=2 k$, where $k$ is odd. We have

(a) $16 \| 17^{k}-1$ since $k$ is odd. Also, $17^{k}-1$ is not divisible by $3,5,7,11,13,23,29,31,37$ and 41 , since $k$ is odd; not divisible by 17 trivially.

(b) $19 \mid 17^{k}-1$ implies $9 \mid k$. This implies that $17^{9}-1 \mid 17^{k}-1$. Also, $17^{9}-1=2^{4} .19 .307 .1270657$. Hence 19,307 and 1270657 divide $\frac{17^{k}-1}{16} \mid \sigma^{* *}\left(17^{c}\right)$; from $(3.8 b)$, it follows that these three prime factors divide $w$. Since $w$ has at most three prime factors, from $(3.8 a)$, we have $n=2^{6} .7^{2} \cdot 17^{c} .5^{d} .19^{e} .(307)^{f} .(1270657)^{g}$ so that

$$
3=\frac{\sigma^{* *}(n)}{n}<\frac{119}{64} \cdot \frac{50}{49} \cdot \frac{17}{16} \cdot \frac{5}{4} \cdot \frac{19}{18} \cdot \frac{307}{306} \cdot \frac{1270657}{1270656}=2.668567854<3,
$$

a contradiction. Hence $19 \nmid 17^{k}-1$.

Thus $\frac{17^{k}-1}{16}$ is odd and not divisible by any of the primes from 3 to 41 . We now prove that $\frac{17^{k}-1}{16}>1$ or $k>1$.

Assume that $k=1$ so that $c=2$. We have $\sigma^{* *}\left(17^{2}\right)=290$. Taking $c=2$ in $(3.8 b)$, we get after simplification

$$
3 \cdot 2^{4} \cdot 7 \cdot 17 \cdot 5^{d-2} \cdot w=29 \cdot \sigma^{* *}\left(5^{d}\right) \cdot \sigma^{* *}(w),
$$

so that $29 \mid w$. Let $w=29^{e} . w^{\prime}$. From $(3.8 a)$ and (3.18), we obtain

$$
n=2^{6} \cdot 7^{2} \cdot 17^{2} \cdot 5^{d} \cdot 29^{e} \cdot w^{\prime},
$$

and

$$
3 \cdot 2^{4} \cdot 7 \cdot 17 \cdot 5^{d-2} \cdot 29^{e-1} \cdot w^{\prime}=\sigma^{* *}\left(5^{d}\right) \cdot \sigma^{* *}\left(29^{e}\right) \cdot \sigma^{* *}\left(w^{\prime}\right),
$$

where $w^{\prime}$ has at most two odd prime factors.

If $p_{1}$ and $p_{2}$ denote these two prime factors of $w^{\prime}$, then it follows from $(3.18 b)$ that $p_{1} \geq 11$ and $p_{2} \geq 13$. Also, $n=2^{6} \cdot 7^{2} \cdot 17^{2} \cdot 5^{d} \cdot 29^{e} \cdot p_{1}^{f} \cdot p_{2}^{g}$. We have

$$
3=\frac{\sigma^{* *}(n)}{n}<\frac{119}{64} \cdot \frac{50}{49} \cdot \frac{290}{289} \cdot \frac{5}{4} \cdot \frac{29}{28} \cdot \frac{11}{10} \cdot \frac{13}{12}=2.937283312<3,
$$

a contradiction. 
Hence $k=1$ is not admissible. We may assume that $k \geq 3$, since $k$ is odd. Thus $\frac{17^{k}-1}{16}$, odd and not divisible by any prime from 3 to 41 . Let $p \mid \frac{17^{k}-1}{16}$. Then $p \geq 43$.

We now consider the factor $17^{k+1}+1$, where $k$ is odd and $\geq 3$.

(c) $17^{k+1}+1$ is not divisible by $3,7,11,13,23$ and 31 since $k+1$ is even; also, $2 \| 17^{k+1}+1$.

(d) $19 \nmid 17^{t}+1$ for any positive integer $t$. In particular, $19 \nmid 17^{k+1}+1$.

(e) $37 \mid 17^{k+1}+1 \Longleftrightarrow k+1=18 u$, where $u$ is odd; this implies that $17^{8}+1 \mid 17^{k+1}+1$. Also, $17^{8}+1=2.5 .29 .37 .109 .181 .2089 .83233 .382069$. Hence $17^{k+1}+1$ is divisible by seven odd prime factors $\geq 29$ and from $(3.8 b)$, these seven prime factors divide $w$. This contradicts the fact that $w$ has no more than three odd prime factors. This proves that $37 \nmid 17^{k+1}+1$.

(f) $41 \mid 17^{k+1}+1 \Longrightarrow k+1=20 u$; This implies that $17^{20}+1 \mid 17^{k+1}+1$. Also, $17^{20}+1=2 \cdot p_{1} \cdot p_{2} \cdot p_{3}$, where $p_{1}=41, p_{2}=41761$ and $p_{3}=1186844128302568601$. Hence $17^{k+1}+1 \mid \sigma^{* *}\left(17^{c}\right)$ is divisible by $p_{1}, p_{2}$ and $p_{3}$. From $(3.8 b)$ it follows that these three primes divide $w$. We have already shown that $p \mid w$, where $p \mid \frac{17^{k}-1}{16}$ and $p \geq 43$. Thus $w$ is divisible by four odd primes $p, p_{1}, p_{2}$ and $p_{3}$. This is not possible. Hence $41 \nmid 17^{k+1}+1$.

(g) We may note that $5\left|17^{k+1}+1 \Longleftrightarrow 29\right| 17^{k+1}+1 \Longleftrightarrow k+1=2 u$.

Suppose that $5 \nmid 17^{k+1}+1$. Then $29 \nmid 17^{k+1}+1$. From (c)-(f) above, it follows that $\frac{17^{k+1}+1}{2}$ is odd, $>1$ and not divisible by any prime from 3 to 41 . If $q \mid \frac{17^{k+1}+1}{2}$, then from (3.8b) it follows that $q \mid w$ and $q \geq 43$.

Suppose that $5 \mid 17^{k+1}+1$ so that $29 \mid 17^{k+1}+1$. Let us assume that $\frac{17^{k+1}+1}{2}=5^{\alpha} \cdot 29^{\beta}$, where $\alpha$ and $\beta$ are positive integers. If $\alpha \geq 2$, then $5^{2} \mid 17^{k+1}+1$. But this is if and only if $k+1=10 u$; in such a case $17^{10}+1 \mid 17^{k+1}+1$. Also, $17^{10}+1=2.5^{2} .29 .21881 .63541$. Hence $21881 \mid \frac{17^{k+1}+1}{2}=5^{\alpha} \cdot 29^{\beta}$. This is obviously false. Hence $\alpha=1$.

Similarly, if $\beta \geq 2,29^{2} \mid 17^{k+1}+1$; this is if and only if $k+1=58 u$ so that $17^{58}+1 \mid 17^{k+1}+1$. Also, $17^{58}+1=2.5 .4908077 . P$, where

$$
P=5627688836691687811685586936872121257317104508544673081805033 .
$$

In particular, $4908077 \mid \frac{17^{k+1}+1}{2}=5^{\alpha} .29^{\beta}$. But this cannot happen. Hence $\beta=1$.

Thus $\frac{17^{k+1}+1}{2}=5.29$ or $17^{k+1}=289$ so that $k+1=2$ or $k=1$. But $k \geq 3$. This contradiction proves that $\frac{17^{k+1}+1}{2}$ must be divisible by a prime $q \neq 5$ and 29 . It now follows that $\frac{17^{k+1}+1}{2}$ is divisible by an odd prime $q$ not in $[3,41]$; also, since $q \mid \sigma^{* *}\left(17^{c}\right)$, from $(3.18 b)$, we have $q \mid w$. The primes $p$ and $q$ are different since they divide $\frac{17^{k}-1}{16}$ and $17^{k+1}+1$ respectively which are relatively prime. As mentioned in the beginning of the case $b=2, d \geq 4,3 \nmid n$ we obtain a contradiction.

The case $b=2$ is complete. The proof of (c) of Theorem 3.1 is complete. 
Proof of (a) of Theorem 3.1.

Case $b \geq 3$. We return to the equations $(3.1 a)$ and $(3.1 b)$, where $b \geq 3$. We claim that $n$ in (3.1a) cannot be a bi-unitary triperfect number. On the contrary we assume that $n$ in $(3.1 a)$ is a bi-unitary triperfect number and obtain a contradiction.

Case $b \geq 3$ with $3 \mid n$. From $(3.1 a), 3 \mid v$. Let $v=3^{d} u$, where $(u, 2.3 .7 .17)=1$. Substituting $v=3^{d} u$ in $(3.1 a)$ and $(3.1 b)$, we obtain

$$
n=2^{6} \cdot 7^{b} \cdot 17^{c} \cdot 3^{d} \cdot u, \quad(b \geq 3)
$$

and

$$
2^{6} \cdot 3^{d+1} \cdot 7^{b-1} \cdot 17^{c-1} \cdot u=\sigma^{* *}\left(7^{b}\right) \cdot \sigma^{* *}\left(17^{c}\right) \cdot \sigma^{* *}\left(3^{d}\right) \cdot \sigma^{* *}(u),
$$

where $u$ has at most three odd prime factors.

By Lemma 2.1, since $b \geq 3, \frac{\sigma^{* *}\left(7^{b}\right)}{7^{b}}>\frac{2752}{2401}$. Also, $\frac{\sigma^{* *}\left(17^{c}\right)}{17^{c}} \geq \frac{88452}{83521}$ when $c \geq 3$ and $\frac{\sigma^{* *}\left(3^{d}\right)}{3^{d}} \geq \frac{112}{81}$ when $d \geq 3$. Hence if $c \geq 3$ and $d \geq 3$, from (3.19a), we get

$$
3=\frac{\sigma^{* *}(n)}{n} \geq \frac{119}{64} \cdot \frac{2752}{2401} \cdot \frac{88452}{83521} \cdot \frac{112}{81}=3.120816493>3
$$

a contradiction.

Hence when $c \geq 3$, then $d=1$ or $d=2$. Let $c \geq 3$.

If $d=1$, from $(3.19 a),(d=1)$, we have

$$
3=\frac{\sigma^{* *}(n)}{n} \geq \frac{119}{64} \cdot \frac{2752}{2401} \cdot \frac{88452}{83521} \cdot \frac{4}{3}=3.009358761>3
$$

a contradiction.

Let $d=2$. Taking $d=2$ in $(3.19 b)$, since $\sigma^{* *}(9)=10$, it follows that $5 \mid u$. Let $u=5^{e} . w$. Using this in $(3.19 a)$ and $(3.19 b)$, we get,

$$
n=2^{6} \cdot 7^{b} \cdot 17^{c} \cdot 3^{2} \cdot 5^{e} \cdot w, \quad(b \geq 3, c \geq 3)
$$

and

$$
2^{5} \cdot 3^{3} \cdot 7^{b-1} \cdot 17^{c-1} \cdot 5^{e-1} \cdot w=\sigma^{* *}\left(7^{b}\right) \cdot \sigma^{* *}\left(17^{c}\right) \cdot \sigma^{* *}\left(5^{e}\right) \cdot \sigma^{* *}(w),
$$

where $w$ has at most two odd prime factors.

We have $\frac{\sigma^{* *}\left(5^{e}\right)}{5^{e}} \geq \frac{756}{625}$ for $e \geq 3$. Hence from $(3.20 a)$, for $e \geq 3$,

$$
3=\frac{\sigma^{* *}(n)}{n} \geq \frac{119}{64} \cdot \frac{2752}{2401} \cdot \frac{88452}{83521} \cdot \frac{10}{9} \cdot \frac{756}{625}=3.033433631>3,
$$

a contradiction.

Hence $e=1$ or $e=2$.

If $e=1$, we have from $(3.20 a)$,

$$
3=\frac{\sigma^{* *}(n)}{n} \geq \frac{119}{64} \cdot \frac{2752}{2401} \cdot \frac{88452}{83521} \cdot \frac{10}{9} \cdot \frac{6}{5}=3.009358761>3,
$$

a contradiction. 
Let $e=2(c \geq 3, d=2)$. Since $\sigma^{* *}\left(5^{2}\right)=26$, taking $e=2$ in $(3.20 b)$, we obtain

$$
2^{5} \cdot 3^{3} \cdot 7^{b-1} \cdot 17^{c-1} \cdot 5^{e-1} \cdot w=26 \cdot \sigma^{* *}\left(7^{b}\right) \cdot \sigma^{* *}\left(17^{c}\right) \cdot \sigma^{* *}(w)
$$

or

$$
2^{4} \cdot 3^{3} \cdot 7^{b-1} \cdot 17^{c-1} \cdot 5^{e-1} \cdot w=13 \cdot \sigma^{* *}\left(7^{b}\right) \cdot \sigma^{* *}\left(17^{c}\right) \cdot \sigma^{* *}(w)
$$

from this equation it follows that $13 \mid w$. Let $w=13^{f} . w^{\prime}$. Now from $(3.20 a)$ and $(3.20 c)$, we obtain

$$
n=2^{6} \cdot 7^{b} \cdot 17^{c} \cdot 3^{2} \cdot 5^{2} \cdot 13^{f} \cdot w^{\prime}, \quad(b \geq 3, c \geq 3)
$$

and

$$
2^{4} \cdot 3^{3} \cdot 7^{b-1} \cdot 17^{c-1} \cdot 5 \cdot 13^{f-1} \cdot w^{\prime}=\sigma^{* *}\left(7^{b}\right) \cdot \sigma^{* *}\left(17^{c}\right) \cdot \sigma^{* *}\left(13^{f}\right) \cdot \sigma^{* *}\left(w^{\prime}\right)
$$

where $w^{\prime}$ has no more than one odd prime factor.

By examining the factors of $\sigma^{* *}\left(7^{b}\right)$ we show that if $b$ is odd or $4 \mid b$, then we obtain a contradiction. If $b=2 k$, where $k$ is odd, we prove that $\frac{7^{k}-1}{6}$ is divisible by a prime $p \geq 29$. From (3.21b), $p \mid w^{\prime}$ and so $w=p^{g}$. So from $(3.21 a)$, we have $n=2^{6} \cdot 7^{b} \cdot 17^{c} \cdot 3^{2} \cdot 5^{2} \cdot 13^{f} \cdot p^{g}$; hence

$$
3=\frac{\sigma^{* *}(n)}{n}<\frac{119}{64} \cdot \frac{7}{6} \cdot \frac{17}{16} \cdot \frac{10}{9} \cdot \frac{26}{25} \cdot \frac{13}{12} \cdot \frac{29}{28}=2.988378605<3,
$$

a contradiction.

We now justify the above.

If $b$ is odd or $4 \mid b$, we have $8 \mid \sigma^{* *}\left(7^{b}\right)$. From $(3.21 b)$, we find that in this case, $2^{5}$ divides its right-hand side but its left-hand side is unitarily divisible by $2^{4}$. This is a contradiction.

In what follows we will be using several results on the divisibility of $7^{k}-1$ by various primes. We refer to Appendix $C$ of [2] for these results.

Let $b=2 k$, where $k$ is odd. Since $b \geq 3$, we have $k \geq 3$.

(a) $2 \| 7^{k}-1$ since $k$ is odd; and $3 \mid 7^{k}-1$.

(b) $7^{k}-1$ is not divisible by $5,11,13,17$ and 23 , since $k$ is odd; trivially not divisible by 7 .

(c) Assume $27 \mid 7^{k}-1$. This implies $9 \mid k$ and so $7^{9}-1 \mid 7^{k}-1$. Also, $7^{9}-1=2.3^{3} .19 .37 .1063$. It follows that $\frac{7^{k}-1}{6}$, a factor of $\sigma^{* *}\left(7^{b}\right)$, is divisible by 19,37 and 1063 . From $(3.21 b)$, these three primes divide $w^{\prime}$. But $w^{\prime}$ is divisible at most by one odd prime factor. Hence $27 \nmid 7^{k}-1$.

(d) We note that $9\left|7^{k}-1 \Longleftrightarrow 19\right| 7^{k}-1 \Longleftrightarrow 3 \mid k$. Hence if $9 \nmid 7^{k}-1$ then $19 \nmid 7^{k}-1$; in this case $\frac{7^{k}-1}{6}$ is not divisible by 3 and 19 . Thus from (a) and (b), $\frac{7^{k}-1}{6}>1$, odd and not divisible by $3,5,7,11,17,19$ and 23 . Hence if $p \mid \frac{7^{k}-1}{6}$, then from $(3.21 b), p \mid w^{\prime}$ and $p \geq 29$.

Suppose that $9 \mid 7^{k}-1$ so that $19 \mid 7^{k}-1$. By (c), $9 \| 7^{k}-1$. Then $\frac{7^{k}-1}{18}$ is odd and $>1$; also not divisible by 3 . Suppose $\frac{7^{k}-1}{18}$ is divisible by 19 alone so that $\frac{7^{k}-1}{18}=19^{\alpha}$. If $\alpha \geq 2$, then $19^{2} \mid 7^{k}-1$; this is if and only if $57 \mid k$ and so $19 \mid k$. But $419\left|7^{19}-1\right| 7^{k}-1$. Hence 
$419 \mid \frac{7^{k}-1}{18}=19^{\alpha}$ which is impossible. Hence $\alpha=1$ and so $\frac{7^{k}-1}{18}=19$ or $k=3$. Hence $b=6$.

We now prove that $b=6$ is not admissible. We have $\sigma^{* *}\left(7^{6}\right)=2.3 .19 .1201$. Taking $b=6$ in $(3.21 b)$, we see that 19 and 1201 divide $w^{\prime}$. But $w^{\prime}$ has at most one odd prime factor. This proves that $b=6$ is not possible. Hence $\frac{7^{k}-1}{18}$ must be divisible by an odd prime say $p \neq 1$. It follows that $p \notin\{3,5,7,11,17,19,23\}$. From $(3.21 b), p \mid w^{\prime}$ and $p \geq 29$.

The case $b \geq 3, c \geq 3,3 \mid n$ is complete.

We may assume that $b \geq 3,3 \mid n$ and $c=1$ or $c=2$. We return to (3.19a) and (3.19b).

Let $c=1$. Since $\sigma^{* *}(17)=18$, taking $c=1$ in $(3.19 a)$ and $(3.19 b)$, we get

$$
n=2^{6} \cdot 7^{b} \cdot 17.3^{d} \cdot u, \quad(b \geq 3)
$$

and

$$
2^{5} \cdot 3^{d-1} \cdot 7^{b-1} \cdot u=\sigma^{* *}\left(7^{b}\right) \cdot \sigma^{* *}\left(3^{d}\right) \cdot \sigma^{* *}(u),
$$

where $u$ has at most three odd prime factors.

From $(3.22 a)$, we have for $d \geq 3$,

$$
3=\frac{\sigma^{* *}(n)}{n} \geq \frac{119}{64} \cdot \frac{2752}{2401} \cdot \frac{18}{17} \cdot \frac{112}{81}=3.120181406>3,
$$

a contradiction.

Hence $d=1$ or $d=2$.

If $d=1$, then $n=2^{6} \cdot 7^{b} \cdot 17.3 . u$ and so we have

$$
3=\frac{\sigma^{* *}(n)}{n} \geq \frac{119}{64} \cdot \frac{2752}{2401} \cdot \frac{18}{17} \cdot \frac{4}{3}=3.008746>3,
$$

a contradiction.

Let $d=2$. Since $\sigma^{* *}\left(3^{2}\right)=10$, taking $d=2$ in $(3.22 b)$ we see that $5 \mid u$. Let $u=5^{e} . w$. With this $u$, from $(3.22 a),(d=2)$, and $(3.22 b),(d=2)$, we get

$$
n=2^{6} \cdot 7^{b} \cdot 17 \cdot 3^{2} \cdot 5^{e} \cdot w, \quad(b \geq 3)
$$

and

$$
2^{4} \cdot 3 \cdot 7^{b-1} \cdot 5^{e-1} \cdot w=\sigma^{* *}\left(7^{b}\right) \cdot \sigma^{* *}\left(5^{e}\right) \cdot \sigma^{* *}(w),
$$

where $w$ can have at most two prime factors and $(w, 2.3 .5 .7 .17)=1$.

If $e \geq 3$, from $(3.22 c)$, we have

$$
3=\frac{\sigma^{* *}(n)}{n} \geq \frac{119}{64} \cdot \frac{2752}{2401} \cdot \frac{18}{17} \cdot \frac{10}{9} \cdot \frac{756}{625}=3.032816327>3,
$$

a contradiction. Hence $e=1$ or $e=2$. If $e=1$, we have $n=2^{6} \cdot 7^{b} \cdot 17.3^{2} .5$.w and so

$$
3=\frac{\sigma^{* *}(n)}{n} \geq \frac{119}{64} \cdot \frac{2752}{2401} \cdot \frac{18}{17} \cdot \frac{10}{9} \cdot \frac{6}{5}=3.008746356>3
$$

a contradiction. 
Let $e=2$. Since $\sigma^{* *}\left(5^{2}\right)=26$, taking $e=2$ in $(3.22 d)$, we find that $13 \mid w$. Let $w=13^{f} . w^{\prime}$. From $(3.22 c)$ and $(3.22 d)$, we get

$$
n=2^{6} \cdot 7^{b} \cdot 17 \cdot 3^{2} \cdot 5^{2} \cdot 13^{f} \cdot w^{\prime}, \quad(b \geq 3)
$$

and

$$
2^{3} \cdot 3 \cdot 7^{b-1} \cdot 5 \cdot 13^{f-1} \cdot w^{\prime}=\sigma^{* *}\left(7^{b}\right) \cdot \sigma^{* *}\left(13^{f}\right) \cdot \sigma^{* *}\left(w^{\prime}\right) ;
$$

$w^{\prime}$ has no more than one odd prime factor and $w^{\prime}$ is prime to 2.3.5.7.13.17.

We obtain a contradiction from $(3.23 b)$ by examining the factors of $\sigma^{* *}\left(7^{b}\right)$.

If $b$ is odd or $4 \mid b$, then $8 \mid \sigma^{* *}\left(7^{b}\right)$. Hence the right-hand side of $(3.23 b)$ is divisible by $2^{4}$ while its left-hand side unitarily by $2^{3}$.

We may assume that $b=2 k$, and $k$ is odd; $b \geq 3$ implies $k \geq 3$. We have

$$
\sigma^{* *}\left(7^{b}\right)=\left(\frac{7^{k}-1}{6}\right) \cdot\left(7^{k+1}+1\right) \text {. }
$$

(a) $2 \| 7^{k}-1$.

(b) $3 \| 7^{k}-1$, since 3 is a unitary divisor of the left-hand side of $(3.23 b)$.

(c) $7^{k}-1$ is not divisible by $5,11,13,17$ and 23 since $k$ is odd; not divisible by 7 trivially.

(d) Since $7^{k}-1$ is not divisible by 9 and hence not divisible by 19 .

From (a)-(d), we conclude that $\frac{7^{k}-1}{6}$ is odd, $>1$ and not divisible by any prime from 3 to 23 . Hence if $p \mid \frac{7^{k}-1}{6}$, then from $(3.23 b), p \mid w^{\prime}$ and $p \geq 29$. Hence $w^{\prime}=p^{g}$ and $n=2^{6} \cdot 7^{b} \cdot 17 \cdot 3^{2} \cdot 5^{2} \cdot 13^{f} \cdot p^{g}$. We have

$$
3=\frac{\sigma^{* *}(n)}{n}<\frac{119}{64} \cdot \frac{7}{6} \cdot \frac{18}{17} \cdot \frac{10}{9} \cdot \frac{26}{25} \cdot \frac{13}{12} \cdot \frac{29}{28}=2.978038194<3,
$$

a contradiction.

The case $c=1$ is complete.

Let $c=2$. The relevant equations are $(3.19 a)$ and $(3.19 b)$. Since $\sigma^{* *}\left(17^{2}\right)=290=2.5 .29$, taking $c=2$ in $(3.19 b)$, we find that $u$ is divisible by 5 and 29 . Hence, $u=5^{e} .29^{f} . w$. From $(3.19 a),(c=2)$, and $(3.19 b),(c=2)$, we obtain the following:

$$
n=2^{6} \cdot 7^{b} \cdot 17^{2} \cdot 3^{d} \cdot 5^{e} \cdot 29^{f} \cdot w, \quad(b \geq 3)
$$

and

$$
2^{5} \cdot 3^{d+1} \cdot 7^{b-1} \cdot 17 \cdot 5^{e-1} \cdot 29^{f-1} \cdot w=\sigma^{* *}\left(7^{b}\right) \cdot \sigma^{* *}\left(3^{d}\right) \cdot \sigma^{* *}\left(5^{e}\right) \cdot \sigma^{* *}\left(29^{f}\right) \cdot \sigma^{* *}(w),
$$

$w$ is prime to 2.3.5.7.17.29 and has no more than one prime factor.

From Lemma 2.1, we have $\frac{\sigma^{* *}\left(5^{e}\right)}{5^{e}} \geq \frac{26}{25}$ for all $e \geq 1$. Using $\frac{\sigma^{* *}\left(3^{d}\right)}{3^{d}} \geq \frac{112}{81}$, for $d \geq 3$, from $(3.24 a)$, we have

$$
3=\frac{\sigma^{* *}(n)}{n} \geq \frac{119}{64} \cdot \frac{2752}{2401} \cdot \frac{290}{289} \cdot \frac{112}{81} \cdot \frac{26}{25}=3.075316052>3,
$$

a contradiction.

Hence $d=1$ or $d=2$. 
Let $d=1$. Since $\sigma^{* *}(3)=4$, taking $d=1$ in $(3.24 b)$, we find that $w=1$. Taking $w=1$ in $(3.24 a)$ and $(3.24 b)$, we get

$$
n=2^{6} \cdot 7^{b} \cdot 17^{2} \cdot 3 \cdot 5^{e} \cdot 29^{f}, \quad(b \geq 3)
$$

and

$$
2^{3} \cdot 7^{b-1} \cdot 17 \cdot 3^{2} \cdot 5^{e-1} \cdot 29^{f-1}=\sigma^{* *}\left(7^{b}\right) \cdot \sigma^{* *}\left(5^{e}\right) \cdot \sigma^{* *}\left(29^{f}\right) .
$$

If $e=1$, from $(3.24 c),(e=1)$, we have

$$
3=\frac{\sigma^{* *}(n)}{n} \geq \frac{119}{64} \cdot \frac{2752}{2401} \cdot \frac{290}{289} \cdot \frac{4}{3} \cdot \frac{6}{5}=3.421711542>3,
$$

a contradiction.

Let $e=2$. Since $\sigma^{* *}\left(5^{2}\right)=26$, taking $e=2$ in $(3.24 d)$, we find that 13 divides its left-hand side which is false.

For $e \geq 3$, using $\frac{\sigma^{* *}\left(5^{e}\right)}{5^{e}} \geq \frac{756}{625}$, from $(3.24 c)$ we get

$$
3=\frac{\sigma^{* *}(n)}{n} \geq \frac{119}{64} \cdot \frac{2752}{2401} \cdot \frac{290}{289} \cdot \frac{4}{3} \cdot \frac{756}{625}=3.449085234>3,
$$

a contradiction.

The case $d=1$ is complete.

Let $d=2$. Taking $d=2$ in $(3.24 a)$ and $(3.24 b)$, we get

$$
n=2^{6} \cdot 7^{b} \cdot 17^{2} \cdot 3^{2} \cdot 5^{e} \cdot 29^{f} \cdot w, \quad(b \geq 3)
$$

and

$$
2^{4} \cdot 3^{3} \cdot 7^{b-1} \cdot 17 \cdot 5^{e-2} \cdot 29^{f-1} \cdot w=\sigma^{* *}\left(7^{b}\right) \cdot \sigma^{* *}\left(5^{e}\right) \cdot \sigma^{* *}\left(29^{f}\right) \cdot \sigma^{* *}(w),
$$

where $w$ is prime to 2.3.5.7.17.29 and has no more than one prime factor.

We shall obtain a contradiction by examining the factors of $\sigma^{* *}\left(7^{b}\right)$.

If $b$ is odd or $4 \mid b$, then $8 \mid \sigma^{* *}\left(7^{b}\right)$. This results in imbalance in powers of two between both sides of $(3.24 f)$.

Let $b=2 k$, where $k$ is odd. Since $b \geq 3$, we have $k \geq 3$. Also,

$$
\sigma^{* *}\left(7^{b}\right)=\left(\frac{7^{k}-1}{6}\right) \cdot\left(7^{k+1}+1\right) .
$$

We consider $7^{k+1}+1$, where $k$ is odd.

(a) $2 \| 7^{k+1}+1$ and $3 \nmid 7^{k+1}+1$; trivially not divisible by 7 .

(b) $29 \nmid 7^{t}+1$ for any $t$; in particular $29 \nmid 7^{k+1}+1$.

(c) Suppose that $5 \nmid 7^{k+1}+1$ and $17 \nmid 7^{k+1}+1$. Then from (a) and (b) it is clear that $\frac{7^{k+1}+1}{2}$ is $>1$, odd and every prime factor of it is not in $\{3,5,7,17,29\}$. Hence each prime factor of $\frac{7^{k+1}+1}{2}$ divides $w$ from $(3.24 f)$. 
(d) Suppose that $5 \mid 7^{k+1}+1$ and $17 \nmid 7^{k+1}+1 ; 5 \mid 7^{k+1}+1 \Longrightarrow k+1=2 u$. Hence $7^{2}+1 \mid 7^{k+1}+1$. Thus $5^{2} \mid 7^{k+1}+1$. Assume that $\frac{7^{k+1}+1}{2}=5^{\alpha}$, where $\alpha \geq 2$. If $\alpha \geq 3$, then $5^{3} \mid 7^{k+1}+1$. This is if and only if $k+1=10 u$. Also, $7^{10}+1=2.5^{3} .281 .4021$. It follows that $281\left|\frac{7^{10}+1}{2}\right| \frac{7^{k+1}+1}{2}=5^{\alpha}$ and this is impossible. Hence $\alpha=2$ so that $\frac{7^{k+1}+1}{2}=5^{2}$. Hence $k=1$. But $k \geq 3$. Thus $\frac{7^{k+1}+1}{2}$ is divisible by an odd prime $q \neq 5$. Also, by our assumption $q \neq 17$. Hence from (a) and (b), $q \notin\{3,5,7,17,29\}$. Since $\frac{7^{k+1}+1}{2} \mid \sigma^{* *}\left(7^{b}\right)$, from $(3.24 f), q \mid w$.

(e) Suppose $17 \mid 7^{k+1}+1$ and $5 \nmid 7^{k+1}+1$. From $(3.24 b), 17$ is a unitary divisor of its left-hand side. Since $17\left|7^{k+1}+1\right| \sigma^{* *}\left(7^{b}\right)$ it follows that $17 \| 7^{k+1}+1$. If $7^{k+1}+1$ is divisible by 17 alone, then we must have $\frac{7^{k+1}+1}{2}=17$ or $7^{k+1}=33$ which is not possible. Hence $\frac{7^{k+1}+1}{2}$ which is $>1$ and odd should be divisible by an odd prime $q \neq 17$. By our assumption $q \neq 5$. Hence from (a) and (b), $q \notin\{3,5,7,17,29\}$. From (3.24f), $q \mid w$.

(f) Suppose that $7^{k+1}+1$ is divisible by both 5 and 17 . Then $5^{2} \mid 7^{k+1}+1$ and $17 \| 7^{k+1}+1$. Assume that $5^{3} \mid 7^{k+1}+1$. This is if and only if $k+1=10 u$. Also, $7^{10}+1=2.5^{3} \cdot 281.4021$. Thus 281 and 4021 divide $7^{k+1}+1$ which is a divisor of $\sigma^{* *}\left(7^{b}\right)$. From $(3.24 f)$, it follows that $w$ is divisible by 281 and 4021 . This is not possible. Hence $5^{2} \| 7^{k+1}+1$. Thus $\frac{7^{k+1}+1}{2.5^{2} .17}$ is odd and $>1$. It must be divisible by an odd prime $q$ and $q \notin\{3,5,7,17,29\}$. From $(3.24 f), q \mid w$.

(g) From (a)-(f), it follows that $\frac{7^{k+1}+1}{2}$ is divisible by an odd prime $q \mid w$. Since $w$ has no more than one prime factor, $w=q^{f}$.

We shall now consider $7^{k}-1$ when $k$ is odd. We have

(h) $2 \| 7^{k}-1$ and $3 \mid 7^{k}-1$.

(i) $9 \mid 7^{k}-1$ if and only if $19 \mid 7^{k}-1$ if and only if $3 \mid k$. Suppose $9 \mid 7^{k}-1$. Then $19 \mid 7^{k}-1$. Hence $19 \mid \sigma^{* *}\left(7^{b}\right)$. From $(3.24 f)$, since $w=q^{f}, q=19$. Since $q\left|\frac{7^{k+1}+1}{2}, 19\right| 7^{k}-1,7^{k}-1$ and $\frac{7^{k+1}+1}{2}$ are relatively prime, $q \neq 19$. This proves that $9 \nmid 7^{k}-1$ (as a consequence $\left.3 \| 7^{k}-1\right)$ and so $19 \nmid 7^{k}-1$.

(j) Since $k$ is odd, $7^{k}-1$ is not divisible by 5 and 17 . Also, $29 \mid 7^{k}-1$ if and only if $7 \mid k$. We have $7^{7}-1=2.3 .29 .4733$. It follows from $(3.24 f)$ that $4733 \mid w=q^{f}$. But $q \neq 4733$ since $q$ and 4733 are prime factors of relatively prime factors. Hence $29 \nmid 7^{k}-1$.

(k) Thus $\frac{7^{k}-1}{6}$ is $>1$, odd and not divisible by any prime in $\{3,5,7,17,29\}$. If $p \mid \frac{7^{k}-1}{6}$, then $p$ is an odd prime $\notin\{3,5,7,17,29\}$. From $(3.24 f), p \mid w=q^{f}$. This is not possible since $p \neq q$.

With this contradiction, the case $d=2$ is complete. Also, the case $c=2,3 \mid n$, is complete.

The case $b \geq 3$ with $3 \mid n$ is complete. 
Case $b \geq 3$ with $3 \nmid n$. We return to the equations $(3.1 a)$ and (3.1b). We assume that $b \geq 3$ and $3 \nmid n$. We show that $n$ cannot be a bi-unitary triperfect number. We first settle this when $5 \nmid n$. We examine $\sigma^{* *}\left(17^{c}\right)$ to obtain a contradiction. We distinguish the following cases:

(i) If $c$ is odd or $4 \mid c$, then $9 \mid \sigma^{* *}\left(17^{c}\right)$. From $(3.1 b)$, it follows that $3 \mid v$. But this is not true since $3 \nmid n$ has been assumed.

(ii) Let $c=2 k$, where $k$ is odd.

(a) Then $17^{k}-1$ is not divisible by $3,5,7,11,13,23,29$ and 37 ; trivially not divisible by 17.

(b) Suppose $19 \mid 17^{k}-1$. This implies $9 \mid k$ and as a consequence $17^{9}-1 \mid 17^{k}-1$. We have $17^{9}-1=2.19 .307 .1270657$. Hence all the three odd prime factors of $17^{9}-1$ divide $\frac{17^{k}-1}{6} \mid \sigma^{* *}\left(17^{c}\right)$. From $(3.1 b)$, these three prime factors divide $v$. Since $v$ is divisible by not more than four prime factors, let $p$ denote the possible fourth prime factor. We can assume that $p \geq 11$. Hence $n=2^{6} \cdot 7^{b} \cdot 17^{c} \cdot 19^{d} \cdot 307^{e} .(1270657)^{f} \cdot p^{g}$, so that

$$
3=\frac{\sigma^{* *}(n)}{n}<\frac{119}{64} \cdot \frac{7}{6} \cdot \frac{17}{16} \cdot \frac{19}{18} \cdot \frac{307}{306} \cdot \frac{1270657}{1270656} \cdot \frac{11}{10}=2.953428577<3,
$$

a contradiction. Hence $19 \nmid 17^{k}-1$.

(c) $32 \nmid 17^{k}-1$, since $k$ is odd. Hence $16 \| 17^{k}-1$.

(d) We now prove that $k>1$. Let $k=1$. Then $c=2$. Since $\sigma^{* *}\left(17^{2}\right)=290,5 \mid \sigma^{* *}\left(17^{2}\right)$. Taking $c=2$ in $(3.1 b)$, we find that $5 \mid v$. This is false since $5 \nmid n$ by our assumption. Hence $k \geq 3$.

From (a)-(d), it follows that $\frac{17^{k}-1}{16}>1$, odd and not divisible by any of the primes $3,5,7,11,13,17,19,23,29$ and 37 . Hence $\frac{17^{k}-1}{16}$ must be divisible by a prime $p \geq 41$. Let the other three prime factors of $v$ be $p_{1}, p_{2}$ and $p_{3}$, where $p_{1} \geq 11, p_{2} \geq 13$ and $p_{3} \geq 19$. Hence $n=2^{6} \cdot 7^{b} \cdot 17^{c} \cdot p_{1}^{d} \cdot p_{2}^{e} \cdot p_{3}^{f} \cdot p^{g}$, so that

$$
3=\frac{\sigma^{* *}(n)}{n}<\frac{119}{64} \cdot \frac{7}{6} \cdot \frac{17}{16} \cdot \frac{11}{10} \cdot \frac{13}{12} \cdot \frac{19}{18} \cdot \frac{41}{40}=2.971682922<3,
$$

a contradiction.

Thus $n=2^{6} \cdot 7^{b} \cdot 17^{c} \cdot v(b \geq 3)$ is not a bi-unitary triperfect number if $3 \nmid n$ and $5 \nmid n$.

We prove that $n=2^{6} 7^{b} 17^{c} v$, where $b \geq 3,5 \mid n, 3 \nmid n$ and $(v, 2.3 .7 .17)=1$ cannot be a bi-unitary triperfect number.

We assume the contrary and obtain a contradiction.

Since $5 \mid n$, we can write $v=5^{d} w$, where $(w, 2.3 .5 .7 .17)=1$. Hence

$$
n=2^{6} 7^{b} 17^{c} 5^{d} w, \quad(b \geq 3) .
$$

If $n$ is a bi-unitary triperfect number, then $\sigma^{* *}(n)=3 n$. Hence from $(3.25 a)$ and since $\sigma^{* *}\left(2^{6}\right)=119=7.17$, the equation $\sigma^{* *}(n)=3 n$ on simplification transforms into

$$
3.2^{6} \cdot 7^{b-1} \cdot 17^{c-1} \cdot 5^{d} \cdot w=\sigma^{* *}\left(7^{b}\right) \sigma^{* *}\left(17^{c}\right) \sigma^{* *}\left(5^{d}\right) \sigma^{* *}(w),
$$


where

$w$ cannot have more than three odd prime factors.

It may be noted that $c \geq 2$ can be assumed; $c=1$ implies that $\sigma^{* *}\left(17^{c}\right)=18$ and so 9 divides the left-hand side of $(3.25 b)$. This is not possible since $w$ is prime to 3 .

Trivially an odd prime factor of the left-hand side of (3.25b) divides $w$ if and only if it does not belong to $\{3,5,7,17\}$.

We essentially use the following lemmas (Lemmas 3.1, 3.2 and 3.3) to prove that $n$ given in $(3.25 a)$ cannot be a bi-unitary triperfect number:

Lemma 3.1. Let $n$ be as in (3.25a) with $w=p_{1}^{e} p_{2}^{f} p_{3}^{g}$, where $p_{1}, p_{2}$ and $p_{3}$ are distinct odd primes with $p_{1} \geq 29, p_{2} \geq 1009$ and $p_{3} \geq 1013$ and $e, f$, and $g$ are positive integers. Then $\sigma^{* *}(n)<3 n$. Hence $n$ cannot be a bi-unitary perfect number.

Proof. We have $n=2^{6} 7^{b} 17^{c} 5^{d} p_{1}^{e} p_{2}^{f} p_{3}^{g}$ so that by Lemma 2.1,

$$
\frac{\sigma^{* *}(n)}{n}<\frac{119}{64} \cdot \frac{7}{6} \cdot \frac{17}{16} \cdot \frac{5}{4} \cdot \frac{29}{28} \cdot \frac{1009}{1008} \cdot \frac{1013}{1012}=2.989869702<3 .
$$

Lemma 3.2. Let $n=2^{6} 7^{b} 17^{c} 5^{d} w(b \geq 3)$ be as given in (3.25a).

(I) If $b$ is odd or $4 \mid b$, then $n$ cannot be a bi-unitary triperfect number.

(II) If $b=6$, then $n$ cannot be a bi-unitary triperfect number.

(III) Let $b=2 k$, where $k \geq 5$ is odd. We have

$$
\sigma^{* *}\left(7^{b}\right)=\left(\frac{7^{k}-1}{6}\right) \cdot\left(7^{k+1}+1\right) .
$$

If $n$ is a bi-unitary triperfect number, then:

(A) $\frac{7^{k}-1}{6}$ is divisible by an odd prime $p^{\prime}>2520$ dividing $w$.

(B) $7^{k+1}+1$ is divisible by an odd prime $q^{\prime} \geq 1201$ dividing $w$.

(C) $n$ is not divisible by 11 or 13 or 19 or 23.

Proof. We assume that $n$ is a bi-unitary triperfect number. Then (3.25b) holds.

Proof of $(I)$. Let $b$ be odd. We have

$$
\sigma^{* *}\left(7^{b}\right)=\frac{7^{b+1}-1}{6}=\frac{\left(7^{t}-1\right)\left(7^{t}+1\right)}{6}
$$

where $t=\frac{b+1}{2}$.

(i) Let $t$ be even. Then $48=7^{2}-1 \mid 7^{t}-1$ and 2|| $7^{t}+1$. Hence $16 \mid \frac{\left(7^{t}-1\right)\left(7^{t}+1\right)}{6}=\sigma^{* *}\left(7^{b}\right)$. It follows from $(3.25 b)$ that $2^{6}$ divides its right-hand side, whereas $2^{6}$ unitarily divides its left-hand side. Hence $w=1$ so that from $(3.25 a), n=2^{6} 7^{b} 17^{c} 5^{d}$. We have

$$
3=\frac{\sigma^{* *}(n)}{n}<\frac{119}{64} \cdot \frac{7}{6} \cdot \frac{17}{16} \cdot \frac{5}{4}=2.881062826<3,
$$

a contradiction. 
(ii) Let $t$ be odd. Then $8 \mid 7^{t}+1$ and $2 \| 7^{t}-1$. Hence $8 \mid \frac{\left(7^{t}-1\right)\left(7^{t}+1\right)}{6}=\sigma^{* *}\left(7^{b}\right)$. From $(3.25 b)$, it follows that, $2^{5}$ divides its right-hand side and $2^{6}$ unitarily divides its left-hand side. Hence $w$ cannot have more than one odd prime factor. We obtain a contradiction by showing that $w$ is divisible by two odd prime factors.

We have $8 \mid 7^{t}+1$. If $16 \mid 7^{t}+1$ then since $2 \| 7^{t}-1$, it follows that $16 \mid \sigma^{* *}\left(7^{b}\right)$ and we obtain a contradiction as in (i). So we may assume that $16 \nmid 7^{t}+1$ and hence $8 \| 7^{t}+1$.

Since $t$ is odd, $7^{t}+1$ is not divisible by 3,5 and 17 ; also not divisible by 7 trivially. We have that $\frac{7^{t}+1}{8}$ is odd and $>1$ since $t \geq 2$ as $b \geq 3$. Hence we can find an odd prime $q\left|\frac{7^{t}+1}{8}\right| \sigma^{* *}\left(7^{b}\right)$; also, $q \notin\{3,5,7,17\}$. From $(3.25 b)$, it follows that $q \mid w$.

We now consider the factor $7^{t}-1$ when $t$ is odd.

(a) We have $2 \| 7^{t}-1$ and $3 \mid 7^{t}-1$.

(b) We may note that $9\left|7^{t}-1 \Longleftrightarrow 3\right| t \Longleftrightarrow 19 \mid 7^{t}-1$. Hence $9\left|7^{t}-1 \Longrightarrow 19\right| 7^{t}-1$ so that $19\left|\frac{7^{t}-1}{6}\right| \sigma^{* *}\left(7^{b}\right)$. From $(3.25 b)$, we see that $19 \mid w$. Already $w$ is divisible by $q$. Since $q\left|7^{t}+1,19\right| 7^{t}-1$ and $q$ is odd, $q \neq 19$. Thus $w$ is divisible by two odd primes, whereas it should be divisible by not more than one odd prime. Hence $9 \nmid 7^{t}-1$; also, $19 \nmid 7^{t}-1$ and $3 \| 7^{t}-1$.

(c) Since $t$ is odd, $7^{t}-1$ is not divisible by 5 or 17 ; not divisible by 7 trivially. Thus $\frac{7^{t}-1}{6}$ is odd, $>1$ and not divisible by $3,5,7$ or 17 . Hence we can find an odd prime $p\left|\frac{7^{t}-1}{6}\right| \sigma^{* *}\left(7^{b}\right)$ and $p \notin\{3,5,7,17\}$. From $(3.25 b), p \mid w$. Since $\frac{7^{t}-1}{6}$ and $7^{t}+1$ are relatively prime, we must have $p \neq q$. Hence $w$ is divisible by two odd primes. But this cannot happen.

The proof of (I) when $b$ is odd is complete.

Now let $b=2 k$, where $k$ is even. This is same as $4 \mid b$.

(iii) Since $k$ is even, $8 \mid 7^{k}-1$ and since $k+1$ is odd, $8 \mid 7^{k+1}+1$, so that $32 \mid \sigma^{* *}\left(7^{b}\right)$. It follows that $2^{8}$ divides the right-hand side of $(3.25 b)$, but $2^{6}$ divides its left-hand side unitarily. This is a contradiction.

The proof of (I) is complete.

Proof of $(I I)$. Let $b=6$. Then $\sigma^{* *}\left(7^{6}\right)=\left(\frac{7^{3}-1}{6}\right) \cdot\left(7^{4}+1\right)=2.3 .19 .1201$.

If we assume that $n$ is a bi-unitary triperfect number, taking $b=6$ in $(3.25 b)$ we get,

$$
2^{5} \cdot 7^{5} \cdot 17^{c-1} \cdot 5^{d} \cdot w=19 \cdot 1201 \cdot \sigma^{* *}\left(17^{c}\right) \sigma^{* *}\left(5^{d}\right) \sigma^{* *}(w) .
$$

It follows from $(3.25 d)$ that $w$ is divisible by 19 and 1201. So we can write, $w=19^{e} .(1201)^{f} . w^{\prime}$, where $w^{\prime}$ is prime to 2.3.5.7.17.19.1201. Hence from $(3.25 a)$,

$$
n=2^{6} 7^{6} 17^{c} 5^{d} 19^{e}(1201)^{f} w^{\prime},
$$

and from $(3.25 d)$,

$$
2^{5} \cdot 7^{5} \cdot 17^{c-1} \cdot 5^{d} \cdot 19^{e-1} \cdot(1201)^{f-1} \cdot w^{\prime}=\sigma^{* *}\left(17^{c}\right) \sigma^{* *}\left(5^{d}\right) \sigma^{* *}\left(19^{e}\right) \sigma^{* *}\left((1201)^{f}\right) \sigma^{* *}\left(w^{\prime}\right),
$$


where

$$
w^{\prime} \text { has at most one odd prime factor. }
$$

By examining the factors of $\sigma^{* *}\left(5^{d}\right)$ we arrive at a contradiction to $(3.26 c)$.

If $d$ is odd, then $3 \mid 5^{d+1}-1$. Hence $3\left|\frac{5^{d+1}-1}{4}\right| \sigma^{* *}\left(5^{d}\right)$. It follows from $(3.26 b)$ that its righthand side is divisible by 3 but its left-hand side is not.

Let $d=2 \ell$, so that $\left.\sigma^{* *}\left(5^{d}\right)\right)=\left(\frac{5^{\ell}-1}{4}\right) \cdot\left(5^{\ell+1}+1\right)$.

If $\ell$ is even, then $3 \mid 5^{\ell}-1$ and so $3 \mid \sigma^{* *}\left(5^{d}\right)$. This leads to a contradiction as above.

We may assume that $d=2 \ell$ and $\ell$ is odd.

If $\ell=1$, then $d=2$ and so $\sigma^{* *}\left(5^{d}\right)=26=2.13$. Taking $d=2$ in (3.26b), we infer that $13 \mid w^{\prime}$ and in view of $(3.26 c), w^{\prime}=13^{g}$, say. From (3.26a), we obtain, $n=2^{6} 7^{6} 17^{c} 5^{2} 19^{e}(1201)^{f} 13^{g}$, so that

$$
3=\frac{\sigma^{* *}(n)}{n}<\frac{119}{64} \cdot \frac{7}{6} \cdot \frac{17}{16} \cdot \frac{26}{25} \cdot \frac{19}{18} \cdot \frac{1201}{1200} \cdot \frac{13}{12}=2.743348734<3,
$$

a contradiction.

We may assume that $\ell>1$ so that $\ell \geq 3$ since $\ell$ is odd.

Since $\ell$ is odd, $4 \| 5^{\ell}-1$ and $5^{\ell}-1$ is not divisible by 7 or 17 ; trivially not divisible by 5 .

$19\left|5^{\ell}-1 \Longleftrightarrow 9\right| \ell$; this implies that $5^{9}-1 \mid 5^{\ell}-1$. We have $5^{9}-1=2^{2} .19 .31 .829$. Hence 31 and 829 divide $\frac{5^{\ell}-1}{4} \mid \sigma^{* *}\left(5^{d}\right)$. From $(3.26 b)$ it follows that $w^{\prime}$ is divisible by 31 and 829 ; this is not possible because of $(3.26 c)$. Thus $19 \nmid 5^{\ell}-1$. Also, $1201\left|5^{\ell}-1 \Longleftrightarrow 600\right| \ell$. Since $\ell$ is odd, $1201 \nmid 5^{\ell}-1$.

Thus $\frac{5^{\ell}-1}{4}$ is odd, $>1$ and not divisible by $5,7,17,19$ or 1201 . Let $p\left|\frac{5^{\ell}-1}{4}\right| \sigma^{* *}\left(5^{d}\right)$ so that $p$ is odd and $p \notin\{5,7,17,19,1201\}$. From (3.26b), $p \mid w^{\prime}$.

We now consider the factor $5^{\ell+1}+1$, where $\ell$ is odd. We have $2 \| 5^{\ell+1}+1$ and it is not divisible by 5,7 or $19 ; 17 \mid 5^{\ell+1}+1 \Longleftrightarrow \ell+1=8 u$ ( $u$ odd); this implies that $5^{8}+1 \mid 5^{\ell+1}+1$. Since $11489 \mid 5^{8}+1$, it follows that $\sigma^{* *}\left(5^{d}\right)$ is divisible by 11489 and from $(3.26 b), 11489 \mid w^{\prime}$. Since $\frac{5^{\ell}-1}{4}$ and $5^{\ell+1}+1$ are relatively prime, $p$ and 11489 divide these factors respectively, we must have $p \neq 11489$. Thus $w^{\prime}$ is divisible by two odd primes contradicting (3.26c). Hence $17 \nmid 5^{\ell+1}+1$.

Also, $1201 \mid 5^{\ell+1}+1 \Longleftrightarrow \ell+1=300 u$, where $u$ is odd; in particular, $\ell+1=12 u^{\prime}$, where $u^{\prime}$ is odd. Hence $5^{12}+1 \mid 5^{\ell+1}$, and $5^{12}+1=2.313 .39001$. Hence 313 and 39001 divide $5^{\ell+1}+1 \mid \sigma^{* *}\left(5^{d}\right)$. From (3.26b) we see that $w^{\prime}$ is divisible by these two odd primes contradicting (3.26c). Hence $1201 \nmid 5^{\ell+1}+1$.

Thus if $q \mid \frac{5^{\ell+1}+1}{2}$, then $q$ is odd and $q \notin\{5,7,17,19,1201\}$; hence from $(3.26 b), q \mid w^{\prime}$. Hence $w^{\prime}$ is divisible by two odd primes $p$ and $q, p \neq q$ contradicting (3.26c).

We have proved that when $b=6, n$ in $(3.25 a)$ cannot be a bi-unitary triperfect number.

The proof of (II) is complete.

Proof of $(I I I)(A)$. Let $b=2 k$, where $k \geq 5$ and odd.

We assume that $n$ given in $(3.25 a)$ is a bi-unitary triperfect number and hence $(3.25 b)$ holds. Let

$$
S_{7}^{\prime}=\left\{p \mid 7^{k}-1: p \in[3,2520]-\{3,19,37,1063\} \text { and } \operatorname{ord}_{p} 7 \text { is odd }\right\}
$$


From Lemma 2.4 (a) of [2], it follows that if $S_{7}^{\prime}$ is non-empty, then we can find a prime $p^{\prime}\left|\frac{7^{k}-1}{6}\right| \sigma^{* *}\left(7^{b}\right)$ and $p^{\prime} \geq 2521$; that is, III (A) of Lemma 3.2 holds. Also, from (3.25b), $p^{\prime} \mid w$.

We may assume that $S_{7}^{\prime}$ is empty. Since $p \nmid 7^{k}-1$ if $\operatorname{ord}_{p} 7$ is even, it follows that:

$\left(A_{1}\right) \frac{7^{k}-1}{6}$ is not divisible by any prime in $[3,2520]$ except possibly $3,9,37$ and 1067.

$\left(A_{2}\right)$ We have $3 \mid 7^{k}-1$ and since $k$ is odd, $2 \| 7^{k}-1$. Also, $27 \nmid 7^{k}-1$. If this is not so, then $9\left|\frac{7^{k}-1}{6}\right| \sigma^{* *}\left(7^{b}\right)$ and from $(3.25 b)$ it follows that $3 \mid w$ and this is not true. We settle the divisibility of $7^{k}-1$ by 9 later.

$\left(A_{3}\right)$ We note that $37\left|7^{k}-1 \Longleftrightarrow 9\right| k \Longleftrightarrow 1063 \mid 7^{k}-1$. We assume that $37 \mid 7^{k}-1$. Hence $7^{9}-1 \mid 7^{k}-1$. Also, $7^{9}-1=2.3^{3} .19 .37 .1063$. Hence $3^{2}\left|\frac{7^{9}-1}{6}\right| \frac{7^{k}-1}{6} \mid \sigma^{* *}\left(7^{b}\right)$. From $(3.25 b), 3 \mid w$. This is false. Hence $37 \nmid 7^{k}-1$.

$\left(A_{4}\right)$ We have $9\left|7^{k}-1 \Longleftrightarrow 3\right| k \Longleftrightarrow 19 \mid 7^{k}-1$.

$\left(A_{5}\right)$ Suppose $19 \nmid 7^{k}-1$. Then $9 \nmid 7^{k}-1$ and so $3 \| 7^{k}-1$. Hence from $A_{1}, A_{2}$ and $A_{3}, \frac{7^{k}-1}{6}$ is not divisible by any prime in $[3,2520]$. Since $\frac{7^{k}-1}{6}>1$ and odd, if $p^{\prime}\left|\frac{7^{k}-1}{6}\right| \sigma^{* *}\left(7^{b}\right)$, then $p^{\prime}>2560$ and $p^{\prime} \mid w$ by $(3.25 b)$.

Hence (III)(A) of Lemma 3.2 follows.

$\left(A_{6}\right)$ Suppose that $19 \mid 7^{k}-1$ so that $9 \mid 7^{k}-1$. Hence $9 \| 7^{k}-1$. It follows that $\frac{7^{k}-1}{18}$ is odd, $>1$ and not divisible by 3 . We can show that $\frac{7^{k}-1}{18}$ is not divisible by 19 alone. Hence we can find an odd prime $p^{\prime} \mid \frac{7^{k}-1}{18}$ and $p^{\prime} \neq 19$. We have $p^{\prime}\left|\frac{7^{k}-1}{18}\right| \frac{7^{k}-1}{6} \mid \sigma^{* *}\left(7^{b}\right)$ and it follows $A_{1}$ to $A_{4}$ that $p^{\prime}>2503$. From $(3.25 b)$, it is clear that $p^{\prime} \mid w$.

This completes the proof of (III)(A).

Proof of $(I I I)(B)$ :

$\left(B_{1}\right)$ Let

$$
T_{7}^{\prime}=\left\{q \mid 7^{k+1}+1: q \in[3,1193]-\{5,13,181,193,409\}\right\} \text { and } s=\frac{1}{2} \operatorname{ord}_{q} 7 \text { is even. }
$$

By Lemma 2.4 (b) of [2], if $T_{7}^{\prime}$ is non-empty, then we can find a prime $q^{\prime}\left|\frac{7^{k+1}+1}{2}\right| \sigma^{* *}\left(7^{b}\right)$ and $q^{\prime}>1193$. By (3.25b), it follows that $q^{\prime} \mid w$. This upholds III(B) of Lemma 3.2.

$\left(B_{2}\right)$ We may assume that $T_{7}^{\prime}$ is empty. Since $q \nmid 7^{k+1}+1$ if $s=\frac{1}{2} \operatorname{ord}_{q} 7$ is not even, from $T_{7}^{\prime}=\emptyset$, we can conclude that $\frac{7^{k+1}+1}{2}$ is not divisible by any prime in $[3,1193]$ except possibly $5,13,181,193$ and 409 .

$\left(B_{3}\right)$ We may note that $193\left|7^{k+1}+1 \Longleftrightarrow 12\right| k \Longleftrightarrow 409 \mid 7^{k+1}+1$. Suppose that $193 \mid 7^{k+1}+1$. This implies that $7^{12}+1 \mid 7^{k+1}+1$. Also, $7^{12}+1=2.73 .193 .409 .1201$. Hence $7^{k+1}+1 \mid \sigma^{* *}\left(7^{b}\right)$ is divisible by four odd primes 73,193, 409 and 1201. From (3.25b), these four odd primes divide $w$. This contradicts $(3.25 c)$. Thus $\frac{7^{k+1}+1}{2}$ is not divisible by 193 and 409 . 
$\left(B_{4}\right)$ We note that $13 \mid 7^{k+1}+1$ if and only if $k+1=6 u$ if and only if $181 \mid 7^{k+1}+1$. Assume that $13 \mid 7^{k+1}+1$ so that $181 \mid 7^{k+1}+1$ and $k+1=6 u$. Hence $7^{6}+1 \mid 7^{k+1}+1$. Also, $7^{6}+1=2 \cdot 5^{2} \cdot 13.181$. So, $5^{2} \mid 7^{k+1}+1$. We now show that $5^{3} \nmid 7^{k+1}+1$. We have $5^{3} \mid 7^{k+1}+1$ if and only if $k+1=10 u$; also, $7^{10}+1=2.5^{3} .281 .4021$. Thus $5^{3} \mid 7^{k+1}+1$ implies that 281 and 4021 divide $7^{k+1}+1 \mid \sigma^{* *}\left(7^{b}\right)$. From $(3.25 b)$, it follows that 281 and 4021 are factors of $w$. Already, 13 and 181 are factors of $7^{k+1}+1 \mid \sigma^{* *}\left(7^{b}\right)$ and from $(3.25 b)$, 13 and 181 divide $w$ also. Thus four prime factors divide $w$ contradicting (3.25c). Hence $5^{3} \nmid 7^{k+1}+1$ and so $5^{2} \| 7^{k+1}+1$.

Clearly, $\frac{7^{k+1}+1}{50}$ is odd, $>1$ and not divisible by 5 . We note that $13^{2} \mid 7^{k+1}+1$ if and only if $k+1=78 u$. Hence $13^{2} \mid 7^{k+1}+1$ implies that $7^{78}+1 \mid 7^{k+1}+1$. From Appendix G of [2], we can see that $7^{78}+1$ has more than three prime factors dividing $w$. This cannot happen. Hence $13^{2} \nmid 7^{k+1}+1$ and so $13 \| 7^{k+1}+1$. Further, $181^{2} \mid 7^{k+1}+1$ if and only if $k+1=1068 u$; also, from Appendix G of [2], $7^{1068}+1$ has more than three prime factors dividing $w$. This contradicts $(3.25 c)$. Hence $181^{2} \nmid 7^{k+1}+1$ and so $181 \| 7^{k+1}+1$.

Thus $13 \mid 7^{k+1}+1$ implies that 13 and 181 are unitary divisors of $\frac{7^{k+1}+1}{50}$; if it is divisible by 13 and 181 alone, then we should have $\frac{7^{k+1}+1}{50}=13.181$ and so $k=5$ or $b=10$. We now prove that $b=10$ is not possible.

We have $\sigma^{* *}\left(7^{10}\right)=\left(\frac{7^{5}-1}{6}\right) \cdot\left(7^{6}+1\right)=2.5^{2} \cdot 13 \cdot 181.2801$. Thus $\sigma^{* *}\left(7^{10}\right)$ is divisible by three prime factors dividing $w$ in $(3.25 b)$. From $(3.25 c)$, we have $w=13^{e} .181^{f} .(2801)^{g}$. Taking $b=10$ in $(3.25 a)$ and $(3.25 b)$, we get

$$
n=2^{6} \cdot 7^{10} \cdot 17^{c} \cdot 5^{d} \cdot 13^{e} \cdot 181^{f} \cdot(2801)^{g},
$$

and

$$
\begin{aligned}
& 3.2^{5} \cdot 7^{9} \cdot 17^{c-1} \cdot 5^{d-2} \cdot 13^{e-1} \cdot 181^{f-1} \cdot(2801)^{g-1} \\
& =\sigma^{* *}\left(17^{c}\right) \cdot \sigma^{* *}\left(5^{d}\right) \cdot \sigma^{* *}\left(13^{e}\right) \cdot \sigma^{* *}\left(181^{f}\right) \cdot \sigma^{* *}\left((2801)^{g}\right),
\end{aligned}
$$

where $c \geq 2$ and $d \geq 2$.

We obtain a contradiction by examining the factors of $\sigma^{* *}\left(17^{c}\right)$ in different cases.

If $c$ is odd or $4 \mid c$, then $9 \mid \sigma^{* *}\left(17^{c}\right)$. It follows from (3.27b) that this cannot happen.

Hence we may assume that $c=2 \ell$ and $\ell$ is odd. Since $\ell$ is odd, $17^{\ell}-1$ is not divisible by $3,5,7$ and 13; trivially not divisible by 17 . Also, $17^{t}-1$ is divisible by 32 if and only if $t$ is even; divisible by 181 if and only if $36 \mid t$ and by 2801 if and only if $56 \mid t$. In these cases, all the values of $t$ must be even. Since $\ell$ is odd, $17^{\ell}-1$ is not divisible by 32 or 181 or 2801 . Since $16 \mid 17^{\ell}-1$ and $32 \nmid 17^{\ell}-1$, we have $16 \| 17^{\ell}-1$. Hence $\frac{17^{\ell}-1}{16}$ is odd.

If $\ell=1$, then $c=2$ and $\sigma^{* *}\left(17^{2}\right)=290$. Hence $29 \mid \sigma^{* *}\left(17^{2}\right)$. Taking $c=2$ in $(3.27 b)$, we find that 29 should divide the right-hand side of it. This is is not possible.

Hence $\ell \geq 3$ and so $\frac{17^{\ell}-1}{16}>1$. Thus $\frac{17^{\ell}-1}{16}>1$, odd and not divisible by $3,5,7,13,17$, 181 and 2801 . Since $\frac{17^{\ell}-1}{16}$ is a factor of $\sigma^{* *}\left(17^{c}\right)$, this cannot happen by virtue of $(3.27 b)$. Therefore, $b=10$ is not possible. 
This proves that $\frac{7^{k+1}+1}{50}$ must be divisible by an odd prime $q^{\prime} \notin\{5,13,181\}$. Now $q^{\prime}\left|\frac{7^{k+1}+1}{50}\right| \frac{7^{k+1}+1}{2}$ and we already proved that $\frac{7^{k+1}+1}{2}$ is not divisible by any prime in $[3,1193]-\{5,13,181\}$, it follows that $q^{\prime}>1193$ (or $q^{\prime} \geq 1201$ ).

Thus we proved (III)(B) when $13 \mid 7^{k+1}+1$.

$\left(B_{5}\right)$ Assume that $13 \nmid 7^{k+1}+1$ and hence $181 \nmid 7^{k+1}+1$. If $5 \nmid 7^{k+1}+1$, then none of the primes in $[3,1193]$ is a factor of $\frac{7^{k+1}+1}{2}$ and so every prime factor of it exceeds 1193 . This upholds the statement in (III)(B). Hence we may assume that $5 \mid \frac{7^{k+1}+1}{2}$. This is if and only if $k+1=2 u$; hence $7^{2}+1=50 \mid 7^{k+1}+1$. Thus $5^{2} \mid 7^{k+1}+1$. If $5^{3} \mid 7^{k+1}+1$ then $k+1=10 u$. Hence $7^{10}+1=2.5^{3} .281 .4021$ is a factor of $7^{k+1}+1$. From (3.25b), it follows that $w$ is divisible by 281 and 4021; also, $w$ is divisible by $p^{\prime}>2521$ dividing $\frac{7^{k}-1}{6}$ from (III)(A). Hence from $(3.25 a), n=2^{6} \cdot 7^{b} \cdot 17^{c} \cdot 5^{d} \cdot 281^{e} \cdot(4021)^{f} \cdot p^{g}$, and so

$$
3=\frac{\sigma^{* *}(n)}{n}<\frac{119}{64} \cdot \frac{7}{6} \cdot \frac{17}{16} \cdot \frac{5}{4} \cdot \frac{281}{280} \cdot \frac{4021}{4020} \cdot \frac{2521}{2520}=2.893219225<3,
$$

a contradiction.

Hence $5^{3} \nmid 7^{k+1}+1$ and so $5^{2} \| 7^{k+1}+1$. If $\frac{7^{k+1}+1}{2}$ is divisible by 5 alone, then we must have $\frac{7^{k+1}+1}{2}=5^{2}$ or $k=1$. But $k \geq 5$. This contradiction proves that $\frac{7^{k+1}+1}{2}$ must be divisible by an odd prime $q^{\prime} \neq 5$. By our assumption, $7^{k+1}+1$ is not divisible by 13 and from $B_{1}, B_{2}$ and $B_{3}$, it follows that $q^{\prime}>1193$ and from $(3.25 b), q^{\prime} \mid w$, since $q^{\prime}$ is a factor of $\sigma^{* *}\left(7^{b}\right)$.

Thus the proof of (III)(B) is complete.

Proof of $(I I I)(C)$. Let $n$ be as given in $(3.25 a)$ and $(3.25 b)$. First we observe that $c \geq 3$. When $\overline{c=2, \sigma^{* *}\left(17^{2}\right)}=290$. Taking $c=2$ in $(3.25 b)$, we see that $29 \mid w$. Let $p^{\prime}$ and $q^{\prime}$ be the primes dividing $w$ obtained in (III)(A) and (III)(B), where $p^{\prime} \geq 2521$ and $q^{\prime}>1193$; so, $q^{\prime} \geq 1201$. Now the primes $29, p^{\prime}$ and $q^{\prime}$ satisfy the hypothesis of Lemma 3.1. Hence $n$ cannot be a bi-unitary triperfect number contrary to our assumption. Hence $c \geq 3$.

(i) Suppose that $11 \mid n$. Hence from $(3.25 a), 11 \mid w$. By $(3.25 c), w=11^{e} p^{\prime f} q^{\prime g}$. From $(3.25 a)$ and $(3.25 b)$, we have

$$
n=2^{6} \cdot 7^{b} \cdot 17^{c} \cdot 5^{d} \cdot 11^{e} \cdot p^{\prime f} \cdot q^{\prime g}
$$

and

$$
3.2^{6} \cdot 7^{b-1} \cdot 17^{c-1} \cdot 5^{d} \cdot 11^{e} \cdot p^{\prime f} \cdot q^{\prime g}=\sigma^{* *}\left(7^{b}\right) \sigma^{* *}\left(17^{c}\right) \sigma^{* *}\left(5^{d}\right) \sigma^{* *}\left(11^{e}\right) \sigma^{* *}\left(p^{\prime f}\right) \sigma^{* *}\left(q^{g}\right) .
$$

When $e=1, \sigma^{* *}\left(11^{e}\right)=12$. Hence $4 \mid \sigma^{* *}\left(11^{e}\right)$. From $(3.28 b)$, it follows that $2^{7}$ divides its right-hand side, whereas $2^{6}$ is a unitary divisor of its left-hand side. This is a contradiction. If $e=2, \sigma^{* *}\left(11^{e}\right)=122=2.61$. Taking $e=2$ in $(3.28 b)$, we find that 61 divides its left-hand side but it cannot divide its right-hand side. Hence we may assume that $e \geq 3$. 
Hence, without loss of generality, we can assume that $b \geq 9, c \geq 3$, and $e \geq 3$. By Lemma 2.1, we have

$$
\begin{gathered}
\frac{\sigma^{* *}\left(7^{b}\right)}{7^{b}} \geq \frac{6723200}{5764801} \quad(b \geq 9), \\
\frac{\sigma^{* *}\left(17^{c}\right)}{17^{c}} \geq \frac{88452}{83521} \quad(c \geq 3)
\end{gathered}
$$

and $\frac{\sigma^{* *}\left(11^{e}\right)}{11^{e}} \geq \frac{15984}{14641},(e \geq 3)$. Also, if $d \geq 3$, then $\frac{\sigma^{* *}\left(5^{d}\right)}{5^{d}} \geq \frac{756}{625}$. From these results and $(3.28 a)$, when $d \geq 3$, we obtain

$$
3=\frac{\sigma^{* *}(n)}{n} \geq \frac{119}{64} \cdot \frac{6723200}{5764801} \cdot \frac{88452}{83521} \cdot \frac{756}{625} \cdot \frac{15984}{14641}=3.032684127>3,
$$

a contradiction.

Hence $d=1$ or $d=2$.

If $d=1$, from $(3.28 a)$,

$$
3=\frac{\sigma^{* *}(n)}{n} \geq \frac{119}{64} \cdot \frac{6723200}{5764801} \cdot \frac{88452}{83521} \cdot \frac{6}{5} \cdot \frac{15984}{14641}=3.008615205>3,
$$

a contradiction.

If $d=2, \sigma^{* *}\left(5^{d}\right)=26$. Hence from (3.28b), it follows that 13 divides its right-hand side but it cannot divide its left-hand side.

Hence $11 \nmid n$.

(ii) Suppose $13 \mid n$. Hence $w=13^{e} p^{\prime f} q^{g}$. From (3.25a) and (3.25b), we get

$$
n=2^{6} \cdot 7^{b} \cdot 17^{c} \cdot 5^{d} \cdot 13^{e} \cdot p^{\prime f} \cdot q^{\prime g},
$$

and

$$
3.2^{6} \cdot 7^{b-1} \cdot 17^{c-1} \cdot 5^{d} \cdot 13^{e} \cdot p^{\prime f} \cdot q^{\prime g}=\sigma^{* *}\left(7^{b}\right) \sigma^{* *}\left(17^{c}\right) \sigma^{* *}\left(5^{d}\right) \sigma^{* *}\left(13^{e}\right) \sigma^{* *}\left(p^{\prime f}\right) \sigma^{* *}\left(q^{g}\right) .
$$

By Lemma 2.1, for $d \geq 5, \frac{\sigma^{* *}\left(5^{d}\right)}{5^{d}} \geq \frac{19406}{15625}$ and for $e \geq 3, \frac{\sigma^{* *}\left(13^{e}\right)}{13^{e}} \geq \frac{30772}{28561}$. Since we have $b \geq 9$ and $c \geq 3$, from $(3.29 a)$, we obtain for $d \geq 5$ and $e \geq 3$,

$$
3=\frac{\sigma^{* *}(n)}{n} \geq \frac{119}{64} \cdot \frac{6723200}{5764801} \cdot \frac{88452}{83521} \cdot \frac{19406}{15625} \cdot \frac{30772}{28561}=3.073045463>3,
$$

a contradiction. Thus $d \geq 5$ implies that $e=1$ or $e=2$.

If $d \geq 5$ and $e=1$, from $(3.29 a)$, we get,

$$
3=\frac{\sigma^{* *}(n)}{n} \geq \frac{119}{64} \cdot \frac{6723200}{5764801} \cdot \frac{88452}{83521} \cdot \frac{19406}{15625} \cdot \frac{14}{13}=3.071647353>3,
$$

a contradiction.

Let $d \geq 5$ and $e=2$. Taking $e=2$ in $(3.29 a)$, we get

a contradiction.

$$
3=\frac{\sigma^{* *}(n)}{n}<\frac{119}{64} \cdot \frac{7}{6} \cdot \frac{17}{16} \cdot \frac{5}{4} \cdot \frac{170}{169} \cdot \frac{2521}{2520} \cdot \frac{1201}{1200}=2.901676629<3,
$$

Thus $d \geq 5$ cannot occur. Hence $d$ takes the choices $1,2,3$ and 4 . 
Let $d=1$. Taking $d=1$ in $(3.29 a)$, we get

$$
3=\frac{\sigma^{* *}(n)}{n}<\frac{119}{64} \cdot \frac{7}{6} \cdot \frac{17}{16} \cdot \frac{6}{5} \cdot \frac{13}{12} \cdot \frac{2521}{2520} \cdot \frac{1201}{1200}=2.999992261<3,
$$

a contradiction.

If $d=2$, from $(3.29 a)$, we obtain

$$
3=\frac{\sigma^{* *}(n)}{n}<\frac{119}{64} \cdot \frac{7}{6} \cdot \frac{17}{16} \cdot \frac{26}{25} \cdot \frac{13}{12} \cdot \frac{2521}{2520} \cdot \frac{1201}{1200}=2.599993292<3,
$$

a contradiction. Since $\sigma^{* *}\left(5^{3}\right)=2^{2} .3 .13$ and $\sigma^{* *}\left(5^{4}\right)=2^{2} .3^{2} .7, \sigma^{* *}\left(5^{d}\right)$ is divisible by 4 when $d=3$ or $d=4$. In these cases $2^{7}$ divides the right-hand side of $(3.29 b)$ while $2^{6}$ is a unitary divisor of its left-hand side.

Thus $13 \nmid n$.

(iii) We assume that $19 \mid n$ so that $w=19^{e} p^{\prime f} q^{\prime g}$. From (3.25a) and (3.25b), we have

$$
n=2^{6} \cdot 7^{b} \cdot 17^{c} \cdot 5^{d} \cdot 19^{e} \cdot p^{\prime f} \cdot q^{\prime g},
$$

and

$$
3.2^{6} \cdot 7^{b-1} \cdot 17^{c-1} \cdot 5^{d} \cdot 19^{e} \cdot p^{\prime f} \cdot q^{\prime g}=\sigma^{* *}\left(7^{b}\right) \sigma^{* *}\left(17^{c}\right) \sigma^{* *}\left(5^{d}\right) \sigma^{* *}\left(19^{e}\right) \sigma^{* *}\left(p^{\prime f}\right) \sigma^{* *}\left(q^{g}\right) .
$$

If $d \geq 7$ and $e \geq 3$, from $(3.29 c)$ we obtain (since $b \geq 9, c \geq 3$ )

$$
3=\frac{\sigma^{* *}(n)}{n} \geq \frac{119}{64} \cdot \frac{6723200}{5764801} \cdot \frac{88452}{83521} \cdot \frac{487656}{390625} \cdot \frac{137561}{130321}=3.026252265>3,
$$

a contradiction; in the above we used $(3.28 c),(3.28 d)$,

$$
\frac{\sigma^{* *}\left(5^{d}\right)}{5^{d}} \geq \frac{487656}{390625}(d \geq 7) \text { and } \frac{\sigma^{* *}\left(19^{e}\right)}{19^{e}} \geq \frac{137561}{130321}(e \geq 3) .
$$

Thus $d \geq 7$ implies that $e=1$ or $e=2$.

Let $d \geq 7$. If $e=1$, then $\sigma^{* *}\left(19^{e}\right)=20$. Hence $4 \mid \sigma^{* *}\left(19^{e}\right)$. From $(3.29 d)$ it follows that there is a mismatch in powers of two between two sides of $(3.29 d)$. If $e=2$, then $\sigma^{* *}\left(19^{e}\right)=362=2.181$. Taking $e=2$ in $(3.29 d)$, we see that 181 divides the left-hand side of $(3.29 d)$, which is false.

Hence $d \geq 7$ is not possible so that $1 \leq d \leq 6$.

Taking $d=1$ in $(3.29 c)$, we have $n=2^{6} \cdot 7^{b} \cdot 17^{c} \cdot 5 \cdot 19^{e} \cdot p^{\prime f} \cdot q^{\prime g}$, and so

$$
3=\frac{\sigma^{* *}(n)}{n}<\frac{119}{64} \cdot \frac{7}{6} \cdot \frac{17}{16} \cdot \frac{6}{5} \cdot \frac{19}{18} \cdot \frac{2521}{2520} \cdot \frac{1201}{1200}=2.923069382<3,
$$

a contradiction.

When $d=2, \sigma^{* *}\left(5^{d}\right)=26=2.13$. Taking $d=2$ in $(3.29 d)$, we see that 13 divides the left-hand side of it and this is not possible.

We have $\sigma^{* *}\left(5^{3}\right)=2^{2} .3 .13$ and $\sigma^{* *}\left(5^{4}\right)=2^{2} .3^{2} .7$. Thus if $d=3$ or $d=4,4 \mid \sigma^{* *}\left(5^{d}\right)$; this results in imbalance in the powers of two between both sides of $(3.29 d)$. 
Also, $\sigma^{* *}\left(5^{5}\right)=2.3^{2} .7 .31$ and $\sigma^{* *}\left(5^{6}\right)=2.31 .313$. Hence if $d=5$ or $d=6,31 \mid \sigma^{* *}\left(5^{d}\right)$. From $(3.29 d)$, it follows that 31 divides the left-hand side of it. This is not possible.

Thus $19 \nmid n$.

(iv) We prove that $23 \nmid n$. On the contrary we assume that $23 \mid n$ and obtain a contradiction. Let $23 \mid n$ and hence $w=23^{e} p^{\prime f} q^{\prime g}$. From (3.25a) and (3.25b), we get,

$$
n=2^{6} \cdot 7^{b} \cdot 17^{c} \cdot 5^{d} \cdot 23^{e} \cdot p^{\prime f} \cdot q^{\prime g}, \quad(b \geq 9, c \geq 3)
$$

and

$$
3.2^{6} \cdot 7^{b-1} \cdot 17^{c-1} \cdot 5^{d} \cdot 23^{e} \cdot p^{\prime f} \cdot q^{\prime g}=\sigma^{* *}\left(7^{b}\right) \sigma^{* *}\left(17^{c}\right) \sigma^{* *}\left(5^{d}\right) \sigma^{* *}\left(23^{e}\right) \sigma^{* *}\left(p^{\prime f}\right) \sigma^{* *}\left(q^{\prime g}\right) .
$$

By Lemma 2.1, we have

$$
\begin{aligned}
\frac{\sigma^{* *}\left(7^{b}\right)}{7^{b}} & \geq \frac{6723200}{5764801} \quad(b \geq 9), \\
\frac{\sigma^{* *}\left(17^{c}\right)}{17^{c}} & \geq \frac{25641254}{24137569} \quad(c \geq 5), \\
\frac{\sigma^{* *}\left(5^{d}\right)}{5^{d}} & \geq \frac{487656}{390625} \quad(d \geq 7), \\
\frac{\sigma^{* *}\left(23^{e}\right)}{23^{e}} & \geq \frac{154752626}{148035889} \quad(e \geq 5) .
\end{aligned}
$$

From $(3.30 a)$, we have for $c \geq 5, d \geq 7$, and $e \geq 5$,

$$
3=\frac{\sigma^{* *}(n)}{n} \geq \frac{119}{64} \cdot \frac{6723200}{5764801} \cdot \frac{25641254}{24137569} \cdot \frac{487656}{390625} \cdot \frac{154752626}{148035889}=3.006276895>3,
$$

a contradiction.

Hence $c \geq 5, d \geq 7$ implies $1 \leq e \leq 4$. Assume that $c \geq 5, d \geq 7$.

We have $\sigma^{* *}(23)=24=2^{3} .3, \sigma^{* *}\left(23^{3}\right)=2^{4} .3 .5 .53$ and $\sigma^{* *}\left(23^{4}\right)=2^{6} .3^{3} .13^{2}$. Hence $2^{3} \mid \sigma^{* *}\left(23^{e}\right)$ when $e=1,3,4$. From $(3.30 b)$, this is not possible as in such a case $2^{8}$ divides its right-hand side, whereas its left-hand side is divisible by $2^{6}$ unitarily.

When $e=2, \sigma^{* *}\left(23^{e}\right)=2.5 .53$. Taking $e=2$ in $(3.30 b)$, it follows that 53 should divide its left-hand side. This is not possible.

Thus $c \geq 5, d \geq 7$ cannot hold.

Let $c \geq 5$ and $1 \leq d \leq 6$.

When $d=1$, from $(3.30 a), n=2^{6} \cdot 7^{b} \cdot 17^{c} \cdot 5 \cdot 23^{e} \cdot p^{\prime f} \cdot q^{\prime g}$ and so we have

$$
3=\frac{\sigma^{* *}(n)}{n}<\frac{119}{64} \cdot \frac{7}{6} \cdot \frac{17}{16} \cdot \frac{6}{5} \cdot \frac{23}{22} \cdot \frac{2521}{2520} \cdot \frac{1201}{1200}=2.895097426<3,
$$

a contradiction.

When $d=2,13 \mid \sigma^{* *}\left(5^{d}\right)$; in this case from $(3.30 b), 13$ should divide its left-hand side. This is not possible.

When $d=3$ or $d=4,4 \mid \sigma^{* *}\left(5^{d}\right)$; in these two cases there will be a mismatch of the powers of 2 between its two sides. 
When $d=5$ or $d=6,31 \mid \sigma^{* *}\left(5^{d}\right)$. Hence form (3.30b), 31 should divide its left-hand side. This is not possible.

Hence $c \geq 5$ is not possible. So, we must have $c=3$ or $c=4$ since $c \geq 3$.

Since $\sigma^{* *}\left(17^{3}\right)=2^{2} .3^{2} .5 .29$ and $\sigma^{* *}\left(17^{4}\right)=2^{2} .3^{5} .7 .13,4 \mid \sigma^{* *}\left(17^{c}\right)$ when $c=3$ or $c=4$.

In these cases we obtain a contradiction from $(3.30 b)$ due to the imbalance of the powers of 2 between its two sides.

Thus $23 \nmid n$.

This proves $(\mathrm{III})(\mathrm{C})$ in all the cases.

Lemma 3.3. The number $n=2^{6} 7^{b} 17^{c} v$, where $b \geq 3,5 \mid n, 3 \nmid n$ and $(v, 2.3 .7 .17)=1$ cannot be a bi-unitary triperfect number.

Proof. Since $5 \mid n, n$ is of the form given in (3.25a). Suppose that $n$ is a bi-unitary triperfect number. By (III)(A) and (B) of Lemma 3.2, w is divisible by primes $p^{\prime}>2507$ and $q^{\prime}>1201$. Let us redesignate $p^{\prime}$ and $q^{\prime}$ by $p_{2}$ and $p_{3}$. Since $w$ cannot have more than three odd prime factors, by (III)(C), a possible third prime factor say $p_{1}$ of $w$ will be $\geq 29$. Now, the primes $p_{1}, p_{2}$ and $p_{3}$ satisfy the hypothesis of Lemma 3.1. Hence $n$ cannot be a bi-unitary triperfect number.

This completes the proof of (a) of Theorem 3.1 and also the proof of Theorem 3.1.

\section{Concluding remarks}

Partial results on bi-unitary triperfect numbers divisible unitarily by $2^{7}$ are obtained. We mention one such result: if $n$ is a bi-unitary triperfect number divisible unitarily by $2^{7}$ and $5^{2}$, then $n=44553600$. We could fix bi-unitary triperfect numbers divisible unitarily by $2^{8} ; 57657600$ is the only such number.

\section{References}

[1] Hagis, P., Jr. (1987). Bi-unitary amicable and multiperfect numbers, Fibonacci Quart., 25 (2), 144-150.

[2] Haukkanen, P., \& Sitaramaiah, V. (2020). Bi-unitary multiperfect numbers, I, Notes Number Theory Discrete Math., 26 (1), 93-171.

[3] Haukkanen, P., \& Sitaramaiah, V. (2020). Bi-unitary multiperfect numbers, II, Notes Number Theory Discrete Math., 26 (2), 1-26.

[4] Sándor, J., \& Crstici, P. (2004). Handbook of Number Theory II, Kluwer Academic.

[5] Suryanarayana, D. (1972). The number of bi-unitary divisors of an integer, in The Theory of Arithmetic Functions, Lecture Notes in Mathematics 251: 273-282, New York, SpringerVerlag.

[6] Wall, C. R. (1972). Bi-unitary perfect numbers, Proc. Amer. Math. Soc., 33 (1), 39-42. 